\title{
Electrostatic electron cyclotron harmonic waves observed by the Akebono satellite near the equatorial region of the plasmasphere
}

\author{
A. Shinbori ${ }^{1}$, T. Ono ${ }^{2}$, M. Iizima ${ }^{2}$, A. Kumamoto ${ }^{2}$, S. Shirai ${ }^{2}$, A. Hanaoka ${ }^{2}$, K. Okamoto ${ }^{2}$, M. Ohashi ${ }^{2}$, and H. Oya ${ }^{3}$ \\ ${ }^{1}$ Solar-Terrestrial Environment Laboratory, Nagoya University, Nagoya 464-8601, Japan \\ ${ }^{2}$ Geophysical Institute, Tohoku University, Sendai 980-8578, Japan \\ ${ }^{3}$ Fukui University of Technology, Fukui 910-8505, Japan
}

(Received April 6, 2004; Revised September 16, 2006; Accepted March 21, 2007; Online published June 27, 2007)

\begin{abstract}
Analysis of the plasma wave observation data provided by the plasma waves and sounder experiment (PWS) on board the Akebono satellite frequently reveals the presence of electrostatic electron cyclotron harmonic (ESCH) waves in the low-latitude region (MLAT $<45^{\circ}$ ) of the plasmasphere within an altitude range from about $3000 \mathrm{~km}$ to the apogee of the satellite (initial apogee was 10,500 km). Even at moderate or low geomagnetic activity, intense ESCH waves often appear near the equatorial region of the plasmasphere above the upper hybrid resonance (UHR) frequency at the lowest harmonic number branch of the $f_{Q n} \mathrm{ESCH}$ waves. We identified these plasma waves as the equatorial plasmasphere $f_{Q_{n}}$ waves $\left(\mathrm{EP}-f_{Q_{n}}\right.$ ). The spectra of the EP- $f_{Q_{n}}$ waves are characterized by a narrow band structure and by a strong nature, with a wave intensity that ranges from $3.46 \times 10^{-8}$ to $3.31 \times 10^{-4} \mathrm{~V} / \mathrm{m}$. The maximum intensity is nearly coincident with the upper limit of the PWS receiver in the low-gain mode. Statistical analysis results reveal that the EP- $f_{O_{n}}$ waves are observable in all the local time sectors; however, the occurrence probability shows a clear enhancement in the early morning sector of 01-03 MLT in the plasmasphere. The EP- $f_{Q n}$ wave activities are suppressed within a period of strong magnetic disturbances as well as solar minimum phase. The linear dispersion relation analysis using a two-component plasma model reveals that supra-thermal plasma with the energy of about $750 \mathrm{eV}$ and with a large temperature anisotropy $(\mathrm{A}=\mathrm{T}$-perp/T-parallel $-1>40)$ must be present in order to realize an appearance of a positive growth rate at the observed frequency and propagation angle of the ESCH waves. Since the hot plasma with such a high anisotropy has not been detected, the validity of the present two-component plasma model remains an open question. The occurrence feature of the $\mathrm{ESCH}$ waves showed that there is a constant activation or a constant flow-in of free energy to generate the strong plasma instability of ESCH waves near the post-midnight sector of the plasmasphere. The existence of ESCH waves revealed that the nature of the plasmaspheric plasma is more turbulent and active than has been believed.
\end{abstract}

Key words: Plasmasphere, temperature anisotropy, equatorial region, hot plasma, UHR, plasma parameter, $f_{Q n}$ resonance, the Akebono satellite.

\section{Introduction}

Electrostatic electron cyclotron harmonic (ESCH) waves are common plasma wave phenomena in the ionosphere and the magnetosphere beyond the plasmapause. They have been named " $(n+1 / 2) f c$ emissions" or "odd half harmonic emissions". ESCH waves in space were observed for the first time in the ionospheric plasma by the Alouette-2 topside sounder experiment, which showed that two types of resonance emissions occurred for $n f_{H}<f_{D n}<(n+1) f_{H}$ and $f_{D n}<f_{\text {UHR }}$ (Nelms and Lockwood, 1966; Oya, 1970) or for $n f_{H}<f_{Q n}<(n+1) f_{H}$ and $f_{Q n}>f_{\mathrm{UHR}}$ (Warren and Hagg, 1968). $f_{H}, f_{D n}, f_{Q n}$, and $f_{\mathrm{UHR}}$ denote electron cyclotron frequency, $f_{D n}$ and $f_{Q n}$ resonance frequencies and upper hybrid resonance (UHR) frequency, respectively. Warren and Hagg (1968) verified that the $f_{Q n}$ resonance frequency is almost coincident with the point of zero group velocity $(V g)$ in the plasma wave dispersion relation

Copyright (c) The Society of Geomagnetism and Earth, Planetary and Space Sciences (SGEPSS); The Seismological Society of Japan; The Volcanological Society of Japan; The Geodetic Society of Japan; The Japanese Society for Planetary Sciences; TERRAPUB of the Bernstein mode. Oya (1971) identified that the $f_{D n}$ resonances is coupled with the $f_{Q n}$ resonance waves by the cyclotron interaction of the order of $n=2$. He also showed that a temperature anisotropy generated due to the RF power injection of sounder pulses plays an important role in stimulating the $\mathrm{ESCH}$ waves in the ionospheric plasma.

The global character and generation region of the $\mathrm{ESCH}$ waves of natural origin observed in the outer magnetosphere have been well studied for three decades (see, for example, Kennel et al., 1970; Fredricks, 1971; Young et al., 1973; Lyons, 1974; Ashour-Abdalla and Kennel, 1978; Hubbard and Birmingham, 1978; Curtis and Wu, 1979; Birmingham et al., 1981; Koons et al., 1987; Roeder and Koons, 1989; Oya, 1991; Roeder et al., 1991; Paranicas et al., 1992; Matsumoto and Usui, 1997; LaBelle et al., 1999; Horne et al., 2003). The most common ESCH waves in the magnetosphere are those occurring between $n f_{H}$ frequencies, denoted the $(n+1 / 2) f_{H}$ waves. The ESCH waves of natural origin were first observed by the OGO-5 satellite (Kennel et al., 1970; Fredericks and Scarf, 1973) as narrow-band $\left(\Delta f / f_{H} \approx 1.0\right)$, large-amplitude $(1-10 \mathrm{mV} / \mathrm{m})$ waves that 
appear within a frequency range of $1.25 f_{H}<f<1.75 f_{H}$. Since this frequency interval is centered at $1.5 f_{H}$, these $\mathrm{ESCH}$ waves are often loosely termed the ' $3 / 2 f_{H}$ ' emissions. Kennel et al. (1970) showed that the ESCH waves appear with an occurrence probability of about $60 \%$ when the OGO-5 satellite crossed the magnetic equator regions between $4<L<10$ in the 00-12 MLT sectors. Oya (1975) subsequently determined that the plasma flow in the plasma sheet is based on the Doppler shift effects of $(n+1 / 2) f_{H}$ waves and that there is a plasma flow with an average speed of $100-200 \mathrm{~km} / \mathrm{s}$ near the plasma sheet. Similar $3 / 2$ emissions have also been observed by the $\mathrm{S}^{3}$-A (Explorer 45) (Anderson and Maeda, 1977) and IMP-6 (Shaw and Gurnett, 1975) satellites. On the IMP-6 satellite observations, $3 / 2 f_{H}$ emissions are sporadically observed on nearly every passage of the outer magnetosphere. It has been suggested that these ESCH waves play an important role in the precipitation of 1- to $10-\mathrm{keV}$ electrons into the auroral ionosphere (Lyons, 1974; Ashour-Abdalla and Kennel, 1978). Shaw and Gurnett (1975) identified 'diffuse' band ESCH waves with substantially wider bandwidths $\left(\Delta f<0.5 f_{H}\right)$ and much lower intensities than the narrow-band emissions. They reported that the 'diffuse' band ESCH waves often tend to appear between the electron gyroharmonics and occur just outside the plasmapause. On the active and passive GEOS-1 plasma wave observation data, Christiansen et al. (1978) identified $f_{Q n}$ waves in the magnetospheric plasma. $f_{Q n}$ waves are attributed to an incoherent process involving a near-Maxwellian plasma distribution with a small population of non-thermal high-energy electrons. These researchers stressed that the $(n+1 / 2) f_{H}$ emissions observed below the UHR frequency are likely due to a different mechanism, a view that is in contrast to that of Shaw and Gurnett (1975) who believed that both types of the emissions are basically due to the same mechanism occurring in different magnetospheric regions.

Hubbard and Birmingham (1978) classified all ESCH waves into four types based on the work of Shaw and Gurnett (1975) and their survey of the IMP-6 satellite data. The first type consists of plasma waves which are seen only in a single narrow band between $f_{H}$ and $2 f_{H}$; these can be further subdivided into two kinds: " $3 / 2$ emissions", with a frequency range of $1.2 f_{H}<f<2.0 f_{H}$, and "low $3 / 2$ emissions", with a frequency range of $1.0 f_{H}<f<1.2 f_{H}$. The second type of ESCH waves, which is called "multiharmonic emissions", is similar in structure and intensity but less frequent in occurrence than the first type. These $\mathrm{ESCH}$ waves are characterized so as to occur in all interharmonic intervals $(n+1 / 2) f_{H}<f<(n+1) f_{H}$ for $n=1$ to the large $n$ value. The maximum value exceeds $n=7$. The third type has a considerably broader frequency band and is weaker in intensity than the first and second type of ESCH waves. This type of ESCH waves has been identified as the diffuse band emissions (Shaw and Gurnett, 1975), and they tend to appear within a frequency range from the electron cyclotron frequency to the plasma frequency. The fourth type consists of one narrow band close to the electron plasma frequency $\left(f_{p}\right)$. Because $f_{p}$ is nearly equal to $f_{\mathrm{UHR}}$ in the region of $f_{p} \gg f_{H}$, such as around the magnetic equator near the plasmapause, the fourth type of ESCH waves are often identified as being mixed with intense UHR waves.

The diffuse type of ESCH waves is observed at all magnetic local times, at magnetic latitude up to $45^{\circ}$ off the magnetic equator (Fredericks and Scarf, 1973), and at a wide L-shell range of 4-12 (Roeder and Koons, 1989). Roeder and Koons (1989) also summarized the occurrence character of these ESCH waves. They surveyed the intensity of the $\mathrm{ESCH}$ waves within $10^{\circ}$ of the magnetic equator and found that these plasma waves occurred most frequently between 0300 and 0600 MLT at geocentric distances of 4-8 Re. The highest intensity of the plasma waves appeared almost exclusively between about 2100 and 0600 MLT. In the 03000600 MLT regions, they found that these plasma waves had intensities of greater than $0.012 \mathrm{mV} / \mathrm{m}$ about $60 \%$ of the time and above $0.035 \mathrm{mV} / \mathrm{m}$ about $25 \%$ of the time. Paranicas et al. (1992) studied a statistical survey of diffusetype ESCH waves using the CRRES plasma wave experiment within a period of 8 months. They showed that the diffuse-type ESCH waves with a large amplitude of about $0.1 \mathrm{mV} / \mathrm{m}$ is confined near the equatorial region within a few degrees of magnetic latitude. The ESCH waves are often observed in the post-midnight quadrant of the magnetosphere between $L=3.5$ and $L=7$ below the electron plasma frequency.

In recent years, the capacity of instruments for plasma wave experiments onboard the scientific satellites has significantly improved, and it is now possible to observe plasma waves at high-frequency resolution with high sensitivity and high time resolutions. The plasma wave and sounder instrument (PWS) onboard the Akebono (EXOSD) satellite has discovered new and important plasma wave phenomena generated inside the plasmasphere and especially near the equatorial region within an altitude range from 274 to 10,500 km (Oya et al., 1990; Oya, 1991). One of the most important discoveries of these phenomena is the appearance of ESCH waves inside the plasmasphere associated with the large magnetic storm, these waves have been classified into the diffuse-band-type emissions identified by Hubbard and Birmingham (1978). Other phenomena that have been observed more recently are enhancements of UHR waves near the equatorial region of the plasmasphere. This phenomenon was named the equatorial enhancements of plasma wave turbulence (EPWAT) by Oya et al. (1990) and Oya (1991). An interesting characteristic of the EPWAT phenomena is that they occur in a remarkably concentrated range of geomagnetic latitude and altitude: $0^{\circ} \pm 10^{\circ}$ and $1,000-10,000 \mathrm{~km}$, respectively. On the other hand, long-term observation results with the PWS instrument show that the other type of plasma waves are frequently observed, appearing above the UHR frequency at the $f_{Q n}$ branch of the ESCH waves in low-latitude regions $\left(\right.$ MLAT $<45^{\circ}$ ) of the plasmasphere. The interesting fact is that these active ESCH waves have been almost stably observable in the plasmasphere and inner magnetosphere with a small L-shell $(L<4)$ region where there has been no detailed survey of the plasma waves by the previous magnetospheric satellites.

The aim of the present study is to clarify the generation region, dependence on magnetic local time and altitude of 


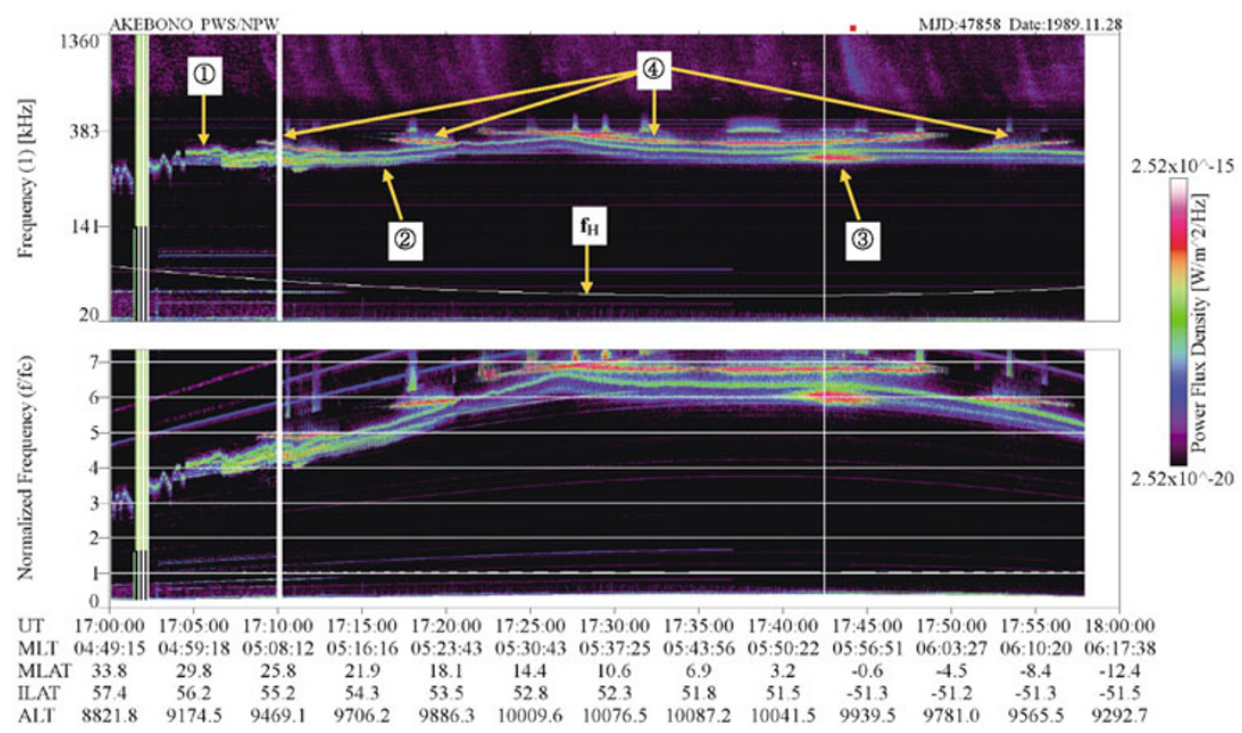

Fig. 1. An example case of plasma wave dynamic spectrum observed inside the low-latitude plasmasphere on November 28, 1989. The upper panel shows plasma wave spectra within a frequency range of $20 \mathrm{kHz}$ to $1.36 \mathrm{MHz}$. The white line gives the local electron cyclotron frequency. The lower panel shows dynamic spectrum normalized by the electron cyclotron frequency. The normalized frequency range of the spectrum is shown up to $n=7$ (cyclotron harmonic number). UT, MLT, MLAT, ILAT, and ALT indicate the universal time, the magnetic local time, the magnetic latitude, the invariant latitude and the altitude of the Akebono satellite, respectively. Plasma wave intensity is indicated by color scale from $2.52 \times 10^{-20}$ $\left(\mathrm{W} / \mathrm{m}^{2} / \mathrm{Hz}\right)$ to $2.52 \times 10^{-15}\left(\mathrm{~W} / \mathrm{m}^{2} / \mathrm{Hz}\right)$. Five line spectra above the UHR frequency show EP- $f_{Q n}$ waves.

the Akebono satellite observation points as well as the characteristics of the occurrence frequency and relation to magnetic and solar activities of the ESCH waves observed in the equatorial plasmasphere region.

\section{Observation Data}

Observations of the Akebono satellite have be ongoing for more than 15 years-since its launch on February 21, 1989 when the satellite was put into a semi-polar orbit with an inclination of $75^{\circ}$ and an initial apogee and perigee of $10,500 \mathrm{~km}$ and $274 \mathrm{~km}$, respectively. In the present study, we have analyzed plasma wave data provided by the PWS instrument in the frequency range of $20 \mathrm{kHz}-5.1$ MHz (Oya et al., 1990) together with magnetic field data obtained by the MGF instrument (Fukunishi et al., 1990) onboard the Akebono satellite. The lower and upper limitations of the PWS detector are $9.49 \times 10^{-18}(\mathrm{~V} / \mathrm{m})^{2} / \mathrm{Hz}$ and $9.47 \times 10^{-13}(\mathrm{~V} / \mathrm{m})^{2} / \mathrm{Hz}$ in the high-gain mode and $9.49 \times 10^{-13}(\mathrm{~V} / \mathrm{m})^{2} / \mathrm{Hz}$ and $9.49 \times 10^{-11}(\mathrm{~V} / \mathrm{m})^{2} / \mathrm{Hz}$ in the low-gain mode, respectively. The bandwidth of the receiver is set as $1 \mathrm{kHz}$ whose receiving frequency is swept with four frequency steps-945 Hz, from $20 \mathrm{kHz}$ to $140 \mathrm{kHz}$; $1.89 \mathrm{kHz}$, from $140 \mathrm{kHz}$ to $382 \mathrm{kHz} ; 7.56 \mathrm{kHz}$, from 382 $\mathrm{kHz}$ to $1360 \mathrm{kHz} ; 30.2 \mathrm{kHz}$, from $1360 \mathrm{kHz}$ to $5120 \mathrm{kHz}$. Therefore, in order to obtain the plasma wave intensity from the power spectrum, we derive this value by using the bandwidth of the receiver. Moreover, in the present analysis, we determined the bandwidth of the observed waves as that frequency range which gives the minimum value of the wave power spectrum. Time resolution of the plasma wave spectra is determined by the swept frequency repetition time of $2 \mathrm{~s}$. On the other hand, we used the archived magnetic field data in order to calculate the electron cyclotron frequency at the location of the Akebono satellite. The time resolution of the magnetic field archived data is $8 \mathrm{~s}$. We also used the Dst and $\mathrm{Kp}$ indices provided by WDC-C2 in Kyoto University in order to clarify geomagnetic activity of the plasmasphere.

\section{Property of EP- $f_{Q n}$ Waves Observed Inside the Plasmasphere}

3.1 EP- $f_{Q n}$ wave phenomena in the low-latitude plasmasphere

3.1.1 An example case of ESCH waves observed on November 28, 1989 Figure 1 shows an example of the dynamic spectrum observed in the plasmasphere on December 11, 1989. The upper panel in Fig. 1 presents the dynamic spectrum from $20 \mathrm{kHz}$ to $1.36 \mathrm{MHz}$ within the time interval of $60 \mathrm{~min}$. The solid curve in the upper panel indicates a variation in electron cyclotron frequency, and the filled square symbol above this panel indicates the passage of the magnetic equator. Plasma wave intensity is indicated by color scale from $2.52 \times 10^{-20} \mathrm{~W} / \mathrm{m}^{2} / \mathrm{Hz}$ as $9.49 \times 10^{-18}(\mathrm{~V} / \mathrm{m})^{2} / \mathrm{Hz}$ to $2.52 \times 10^{-15} \mathrm{~W} / \mathrm{m}^{2} / \mathrm{Hz}$ as $1.02 \times 10^{-13}(\mathrm{~V} / \mathrm{m})^{2} / \mathrm{Hz}$. The lower panel presents the dynamic spectrum normalized by the local electron cyclotron frequency, which is calculated from the magnetic field data obtained by the MGF detector onboard the Akebono satellite. The normalized frequency range of this spectrum is shown up to $n=7$ (the 7th cyclotron harmonic frequency). The time interval is the same as that of the upper panel. UT, MLT, MLAT, ILAT, and ALT below the dynamic spectrum indicate the universal time (UT), the magnetic local time, the magnetic latitude, the invariant latitude and the altitude of the Akebono satellite, respectively.

In this case, the Akebono satellite passed through the low-latitude $\left(-12.4^{\circ}<\right.$ MLAT $\left.<33.8^{\circ}\right)$ and high-altitude $(8,821-10,050 \mathrm{~km})$ region of the dawnside (04:49-06:17 MLT) plasmasphere from the northern to southern hemisphere. The average Dst and Kp indices for this observation time period were $-10 \mathrm{nT}$ and $3+$, respectively. The 
present spectrum is therefore obtained during the moderate geomagnetic condition. Two narrow-banded spectra within a frequency range of $286-367 \mathrm{kHz}$ can be seen in the upper panel in Fig. 1. The upper line spectrum marked by arrow (1) consists of UHR waves, while the lower line spectrum marked by arrow (2) was identified as $Z$-mode waves. Here, we identified the $Z$-mode waves by determining the plasma frequency $\left(f_{p}=\frac{1}{2 \pi} \sqrt{\frac{n e^{2}}{m_{e} \varepsilon_{0}}}\right)$ and cut-off frequency of the $Z$-mode waves from the cold plasma dispersion relation $\left(f_{z-c u t}=-\frac{f_{H}}{2}+\sqrt{\frac{1}{4} f_{H}^{2}+f_{p}^{2}}\right)$, based on the upper limited frequency of the UHR waves $\left(f_{\mathrm{UHR}}=\sqrt{f_{H}^{2}+f_{p}^{2}}\right)$ and the local electron cyclotron frequency $\left(f_{H}=\frac{1}{2 \pi} \frac{e B}{m_{e}}\right)$. Furthermore, the enhancements of the $Z$-mode waves marked by arrow (3) take place at the time interval of 17:40-17:45 UT near the narrow magnetic equatorial $\left(-1.28^{\circ}<\right.$ MLAT $<$ $2.88^{\circ}$ ) region of the plasmasphere. On the other hand, four narrow-band spectra marked by arrows (4) with a large intensity, which appear above the UHR waves within a frequency range of $307-383 \mathrm{kHz}$, are seen at the time intervals of 17:08-17:13, 17:14-17:20, 17:21-17:50, 17:5117:57 and 18:25 (UT). The frequencies of all line spectra are continuously varying in correspondence to the variation in the local electron cyclotron frequency. However, it is clear that the frequency of these plasma waves is strictly higher than the upper cutoff frequency of the UHR waves. In the lower panel, the most remarkable character of the four line spectra is that the upper cutoff frequency of each spectrum is just equal to the frequencies of the electron cyclotron harmonics, namely, $n f_{H}$. The line spectra with large amplitude disappear when the UHR frequency crosses the electron cyclotron harmonic frequencies. The second characteristic which distinguishes these waves is the variations in the frequency, which varies with the similar tendency of the UHR frequency. These characteristics of the four line spectra above the UHR frequency indicate the nature of the ESCH waves. As shown in the following discussion of Section 3.2, the occurrence frequency of the intense ESCH waves strongly depends on both the electron local cyclotron frequency and the plasma parameter $\left(f_{p} / f_{H}\right)$. The frequency of the ESCH waves appears in a range of $(n+1 / 2) f_{H}<f<(n+1) f_{H}$. Furthermore, its occurrence frequency is almost coincident with the $f_{Q n}$ wave frequency at $\mathbf{V} g=0$ (group velocity) belonging to the UHR wave branch. The frequency of the $f_{Q n}$ wave is deduced from the plasma wave dispersion analysis, taking into account a typical electron distribution function of the cold and hot component based on the Cassini (Rymer et al., 2001) and CRRES (Burke et al., 1995) satellite observations. Therefore, from the above discussion on the occurrence frequency of the ESCH waves, it can be concluded that these phenomena are $f_{Q n}$ waves. The present $f_{Q n}$ waves show a different nature from those observed by the sounder experiment in the ionosphere (Warren and Hagg, 1968) and magnetosphere (Christiansen et al., 1978) because the present $f_{Q n}$ waves are strictly limited within the frequency range $(n+1 / 2) f_{H}<f_{Q n}<(n+1) f_{H}$. Therefore, we named this ESCH waves as Equatorial Plasmasphere $f_{Q n}$ waves (EP$\left.f_{Q n}\right)$. The spectra bandwidth of the present EP- $f_{Q n}$ waves

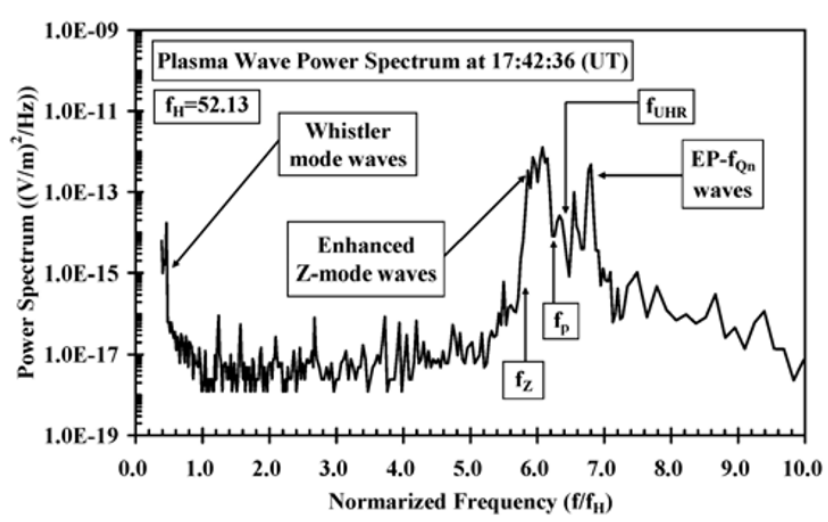

Fig. 2. Power spectrum of plasma waves observed at 17:42:36 (UT) on November 28,1989 . Frequency is normalized by the local electron cyclotron frequency $(52.13 \mathrm{kHz})$. EP- $f_{Q n}$ waves have the large amplitude of $2.4 \times 10^{-10}\left[(\mathrm{~V} / \mathrm{m})^{2} / \mathrm{Hz}\right]$ at $6.8 f_{H}$.

tends to be wider with increasing intensity. It is noted that three continuous lines and sporadic spectra at frequencies of 400,440 , and $480 \mathrm{kHz}$, respectively, and near two band sectors (at $383 \mathrm{kHz}$ ) above the UHR frequency are not of a natural origin but artificial interferences from the instruments onboard the Akebono satellite.

Figure 2 shows a frequency spectrum observed in the high-gain mode at 17:42:36 (UT) on November 28, 1989. In this case, the Akebono satellite was located at $9995.4 \mathrm{~km}$, 05:53 LT, and $1.2^{\circ}$ in the equatorial region of the plasmasphere. The local electron cyclotron frequency is $52.13 \mathrm{kHz}$. The saturation level of the high-gain mode is $9.47 \times 10^{-13}$ $(\mathrm{V} / \mathrm{m})^{2} / \mathrm{Hz}$. In Fig. 2, the power spectrum of the EP- $f_{Q n}$ waves is identified as having a narrow band structure within a frequency range of $6.69 f_{H}<f<6.91 f_{H}$. The peak power spectrum was measured as $4.75 \times 10^{-13}(\mathrm{~V} / \mathrm{m})^{2} / \mathrm{Hz}$ at $6.8 f_{H}$ and corresponds to a wave amplitude of about $2.2 \times 10^{-5} \mathrm{~V} / \mathrm{m}$. The enhancements of the power spectrum with an average value of $4.79 \times 10^{-13}(\mathrm{~V} / \mathrm{m})^{2} / \mathrm{Hz}$ can also be seen in a frequency range from the $Z$-mode cutoff to plasma frequencies $\left(5.76 f_{H}<f<6.25 f_{H}\right)$. The power spectrum corresponds to the wave amplitude of about $1.1 \times 10^{-4} \mathrm{~V} / \mathrm{m}$.

3.1.2 An example case of an ESCH wave observed on December 11, 1989 Figure 3 shows another example of the dynamic spectrum of the EP- $f_{Q n}$ waves observed in the plasmasphere on December 11, 1989. The format of this dynamic spectrum is the same as that of Fig. 1. The time interval is $90 \mathrm{~min}$. In this case, the Akebono satellite passed through the low-latitude $\left(-37.6^{\circ}<\right.$ MLAT $\left.<37.8^{\circ}\right)$ and high-altitude $(6,420-10,050 \mathrm{~km})$ region of the dawnside (03:18-05:30 MLT) plasmasphere from the northern to southern hemisphere. The average Dst and $\mathrm{Kp}$ indices in this time were $-5 \mathrm{nT}$ and 2 , respectively. The results indicate a quiet condition in terms of magnetic activities. In this event also, two narrow-banded spectra of the UHR (marked by arrow (1) and $Z$-mode (marked by arrow (2)) waves can be seen in the entire plasmasphere regions. Furthermore, the phenomena of the $Z$-mode wave enhancements (marked by arrow (3)) take place at 17:53-18:05 (UT) near the magnetic equator $\left(-5.0^{\circ}<\right.$ MLAT $\left.<5.0^{\circ}\right)$ region of the plasmasphere. Within five time intervals of 17:17-17:24, 


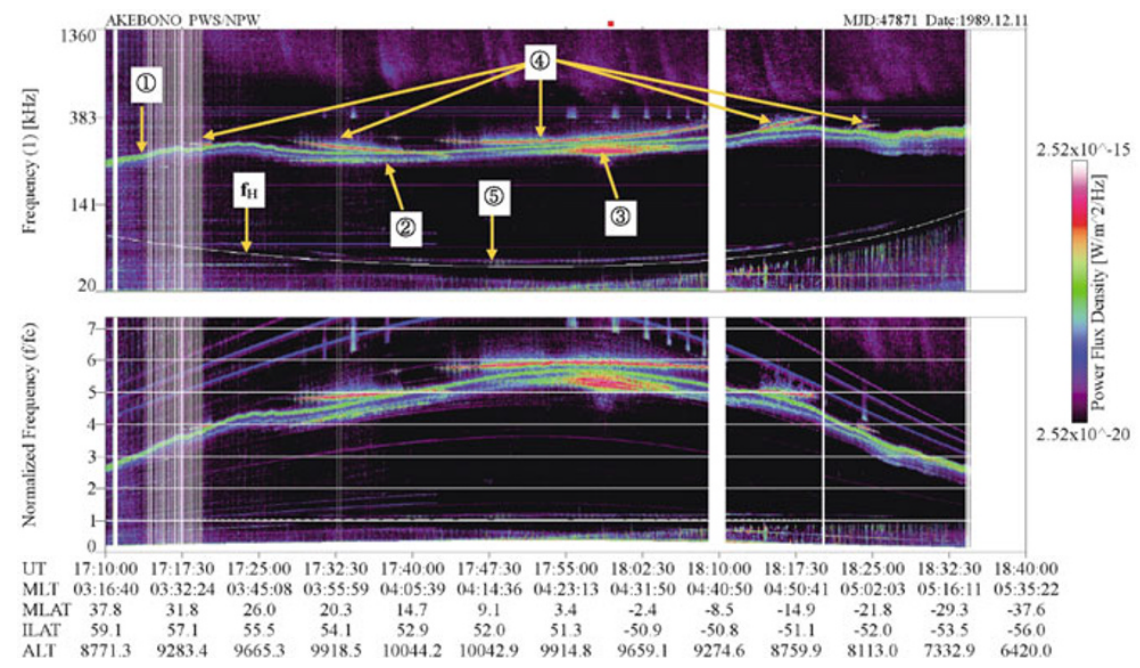

Fig. 3. An example case of plasma wave dynamic spectrum observed inside the low latitude plasmasphere on December 11,1989 . The format is the same as that of Fig. 1. The time interval of the dynamic spectra is $90 \mathrm{~min}$. Another type ESCH waves can be seen just above the electron cyclotron frequency.

17:25-17:39, 17:42-18:10, 18:13-18:20, and 18:23-18:25 (UT), the EP- $f_{Q n}$ waves with intense line spectra (marked by arrows (4)) are also observed above the UHR frequency within a frequency range of $307-383 \mathrm{kHz}$. The character of these line spectra is almost the same as that of the above event. The satellite spin modulation with a period of $4 \mathrm{~s}$ is found in a part of the EP- $f_{Q n}$ wave spectra.

Another type of plasma wave with a continuous narrow band structure (marked by arrow (5)) can be seen just above the electron cyclotron frequency from 17:20 to 18:30 (UT) in the upper panel of Fig. 3. The observed frequency of the plasma waves is closely related to the electron cyclotron frequency. In the lower panel, the plasma waves appear within a frequency range of $1.0 f_{H}<f<1.2 f_{H}$. Based on this relation, it can also be concluded that these plasma waves also have the character of ESCH waves. This kind of the ESCH wave can perhaps be categorized into "low 3/2 emissions" with a frequency range of $1.0 f_{H}<f<1.2 f_{H}$ identified in the magnetosphere by Hubbard and Birmingham (1978). In this case, there can be three continuous or sporadic spectra at a frequency of 400,440 , and $480 \mathrm{kHz}$, and near two band sectors (at $383 \mathrm{kHz}$ ) above the UHR frequency; however, these emissions are not of a natural origin but are actually artificial interference from the instruments onboard the Akebono satellite, as has already been described in the previous event.

Figure 4 shows a frequency spectrum observed at 17:52:00 (UT) on December 11, 1989. In this case, the Akebono satellite was located at $10042.9 \mathrm{~km}, 04: 14 \mathrm{LT}$, and $9.1^{\circ}$ in the plasmasphere, and the PWS instrument was operated in high-gain mode. The electric field intensity range detectable in this mode is $9.48 \times 10^{-18}(\mathrm{~V} / \mathrm{m})^{2} / \mathrm{Hz}$ to $9.47 \times 10^{-13}(\mathrm{~V} / \mathrm{m})^{2} / \mathrm{Hz}$. The local electron cyclotron frequency is $53.53 \mathrm{kHz}$. In Fig. 4, the power spectrum of the low $3 / 2 \mathrm{ESCH}$ waves are identified within a frequency range of $1.01 f_{H}<f<1.23 f_{H}$. The peak intensity of the plasma waves is $2.84 \times 10^{-14}(\mathrm{~V} / \mathrm{m})^{2} / \mathrm{Hz}$ at $1.13 f_{H}$. The power spectrum corresponds to a wave amplitude of about $5.3 \times 10^{-6} \mathrm{~V} / \mathrm{m}$. The power spectrum of the second har-

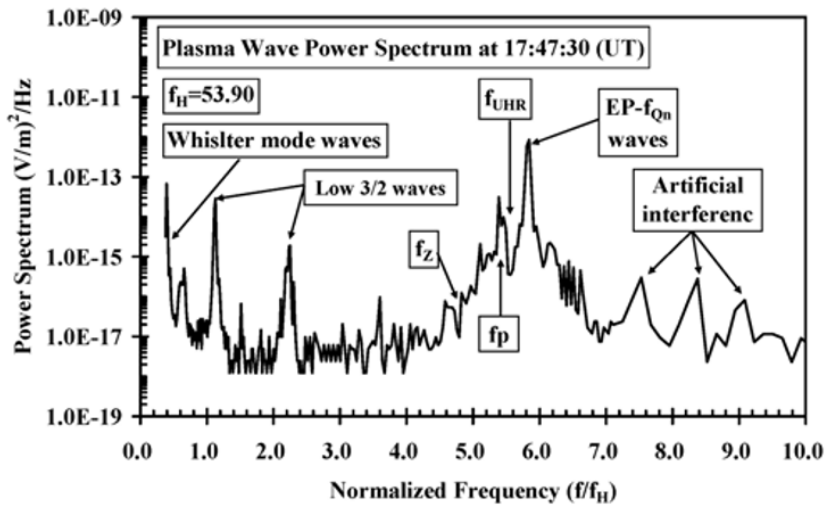

Fig. 4. Power spectrum of plasma waves observed at 17:47:30 (UT) on December 11, 1989. The format is the same as that of Fig. 2. The local electron cyclotron frequency is $53.90 \mathrm{kHz}$. Two peaks can be seen within a frequency range of $1.0 f_{H}<f<3.0 f_{H}$.

monics of the low $3 / 2 \mathrm{ESCH}$ waves is also identified within a frequency range of $2.02 f_{H}<f<2.37 f_{H}$. The peak intensity of the plasma waves is $1.89 \times 10^{-15}(\mathrm{~V} / \mathrm{m})^{2} / \mathrm{Hz}$ at $2.25 f_{H}$, and the power spectrum corresponds to the wave amplitude of about $1.4 \times 10^{-6} \mathrm{~V} / \mathrm{m}$. The frequencies of the fundamental and second harmonic low $3 / 2 \mathrm{ESCH}$ waves at the maximum intensity are $1.13 f_{H}$ and $2.25 f_{H}$, respectively; as such, the fundamental frequency is almost equal to twice that of the second harmonic frequency. On the other hand, the EP- $f_{Q n}$ waves show a narrow-frequency band with the maximum intensity of these plasma waves, which are located above the UHR band. The intensity of the EP- $f_{Q n}$ waves is $8.50 \times 10^{-13}(\mathrm{~V} / \mathrm{m})^{2} / \mathrm{Hz}$ at $5.84 f_{H}$. The wave amplitude is about $2.9 \times 10^{-5} \mathrm{~V} / \mathrm{m}$; this value is less than the upper limit $\left(3.57 \times 10^{-5} \mathrm{~V} / \mathrm{m}\right)$ of the PWS receiver in the high-gain mode. This upper limit value was derived by multiplying the upper value of $8.78 \times 10^{-13}(\mathrm{~V} / \mathrm{m})^{2} / \mathrm{Hz}$ by the frequency resolution $(1 \mathrm{kHz})$ of the PWS receiver. Three spectral peaks which appear within a frequency range of $7.0 f_{H}<f<10 f_{H}$ indicate the artificial interference 


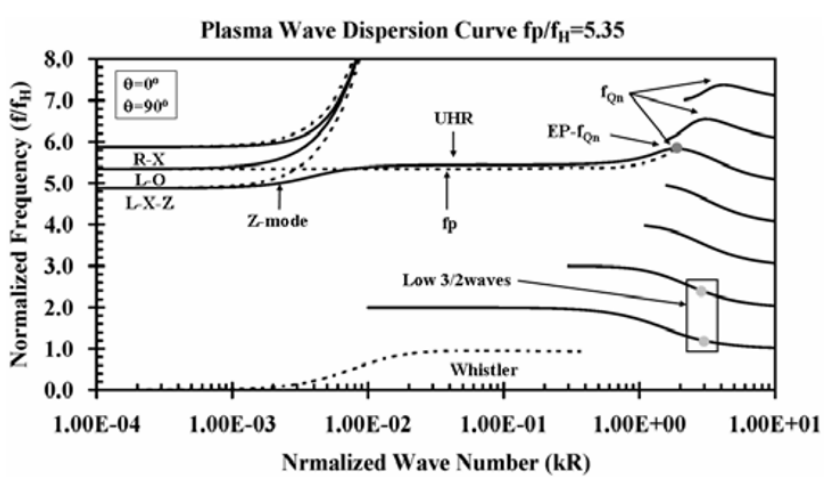

Fig. 5. The dispersion curves for multi-component plasmas calculated in the plasma condition of the passage of the Akebono satellite on the plasmasphere at 17:47:30 on December 11, 1989. The temperatures of the background cold and hot components are $0.5 \mathrm{eV}$ and $750 \mathrm{eV}$, respectively. The wave number $k$ and frequency $f$ are normalized by the electron Lamor radius $R$ and cyclotron frequency, respectively. The curves are drawn for wave normal angles perpendicular $\left(\theta=90^{\circ}\right)$ and parallel $\left(\theta=0^{\circ}\right)$ to the magnetic field.

due to the satellite instruments, as has been described previously.

Figure 5 shows plasma wave dispersion curves corresponding to the plasma parameter $\left(f_{p} / f_{c}=5.35\right)$ of the Akebono satellite passage through the plasmasphere at 17:47:30 (UT) on December 11, 1989. Although we need to be informed of the particle observation in the plasmasphere for the calculation of the dispersion curves, it is unfortunate that the Akebono satellite does not operate the low-energy particle observations obtained from the LEP detector in order to avoid the effect on radiation belt particles. Therefore, instead of using the particle observations by the Akebono satellite in this region, we referred to the Cassini (Rymer et al., 2001) and CRRES (Burke et al., 1995) satellite observations for the background cold and hot plasma distributions in the low $L$-value $(L<3.0)$ region of the plasmasphere, respectively. Both satellite observations suggest that the plasma distribution in this region consists of two components of the cold and hot plasmas. From this result, we adapted the two-component plasma model in calculating the plasma wave dispersion relation. In this plasma model, we assumed that the cold and hot plasma components have a Maxwellian and temperature anisotropy distribution, respectively. The Cassini satellite observations supported the assumption that the background cold plasma distribution in the plasmasphere can be regarded as Maxwellian. On the other hand, from the low-energy electrons observations by the CRRES satellite, Burke et al. (1995) showed that there exists hot component plasma with energy and a perpendicular temperature to the magnetic field of $3 \mathrm{keV}$ and $750 \mathrm{eV}$, respectively, in the inner plasmasphere around $L=2.6$. Moreover, the pitch angle observations of the $\mathrm{keV}$-range electrons in this region indicated the concentration of the electron fluxes around $80-90^{\circ}$ to the ambient magnetic field line. This distribution showed a large temperature anisotropy of $T_{\perp} \gg T_{\|}$. When we calculate the temperature anisotropy index from the pitch angle distribution, it becomes $A=T_{\perp} / T_{\|}-1=\tan ^{2} \alpha_{e q}>32$. Hence, we adapted the temperature anisotropy distribution with $T_{\perp}=750 \mathrm{eV}, T_{\|}=14.7 \mathrm{eV}$, and $A=50$ for the representative hot component in calculating the plasma wave dispersion relation. We will discuss the possibility of this particle distribution in the inner plasmasphere in Section 5.4. On the other hand, the ratio of the hot component to background plasma density (nh/nc) is in a range of about $0.01-$ $0.1 \%$ in the usual plasmasphere region. For the present calculation of the dispersion relation, we used the above ratio of $0.1 \%$. Furthermore, the background plasma temperature is determined from the electron temperature observations obtained from the TED detector (Abe et al., 1990) onboard the Akebono satellite. In this case, the background plasma temperature in the plasmasphere was about $0.5 \mathrm{eV}$. Using these plasma parameters, we calculated the plasma wave dispersion relation shown in Fig. 5. Below the electron plasma frequency $(f p e)$, the electrostatic $(n+1 / 2) f_{H}$ waves can propagate in the backward mode (i.e., the perpendicular group velocity is negative and oppositely directed to the $\mathrm{k}$ vector), while above the fpe, electrostatic waves may propagate in either the forward or backward modes. The maximum frequency in the dispersion curves above fpe for $k R>1.0$ ( $R$ : electron Larmor radius) is well known as the $f_{Q n}$ resonance frequency-which is the maximum frequency lying between the harmonics of the electron cyclotron frequency. The UHR waves shown in Fig. 5 are propagating in the UHR branch that connects the electrostatic waves and electromagnetic plasma waves $(Z$-mode waves) and in the electrostatic branch which connects to the $Z$-mode branch at $\theta=90^{\circ}$. The frequency of almost the plateau region corresponds to fUHR, which lies very close to fpe, since $f p e \gg f c$. These waves are generally believed to be the strongest emissions since their group velocities are very small and thus the waves are possibly amplified strongly. As the observed plasma wave spectra have already been described in Fig. 4, the peak intensity of the EP$f_{Q n}$ waves is located at $5.84 f_{H}$, while the $f_{Q n}$ frequency of the lowest harmonic number branch above the UHR frequency is shown in Fig. 5, which is calculated for multicomponent plasmas of $0.5 \mathrm{eV}$ and $750 \mathrm{eV}$ to give $5.87 f_{H}$. Moreover, the hot component (suprathermal) plasma has a large temperature anisotropy of $A=50$. This frequency is almost in agreement with that of the observed EP- $f_{Q n}$ waves within a frequency accuracy of the PWS instrument $(\delta f / f= \pm 0.03-0.06)$. Therefore, the above result suggests that the velocity distribution of the plasmaspheric plasma within a suprathermal energy range of $\sim 3 \mathrm{keV}$ is basically a temperature anisotropy distribution. Moreover, in the dispersion curves of the $f_{Q n}$ waves, it can be inferred that the observed EP- $f_{Q n}$ wave number is about $1.1 \mathrm{~m}^{-1}$ for the electron Larmor radius of $1.2 \mathrm{~m}$. Here, we calculated the Larmor radius from the cold part of the electron temperature $(0.5 \mathrm{eV})$ and cyclotron frequency $(53.9 \mathrm{kHz})$ at the observation point at 17:47:30 (UT) on December 11, 1989. The occurrence feature of the EP- $f_{Q n}$ waves on the basis of the statistical analysis will be shown in the next section. On the other hand, as shown in the dispersion curves of Fig. 5, the fundamental and second harmonics of "Low 3/2 emissions" observed in the plasmasphere are located at the two lowest $(n+1 / 2) f_{H}$ branches, respectively, in a region of large wave numbers. These normalized wave numbers are about 


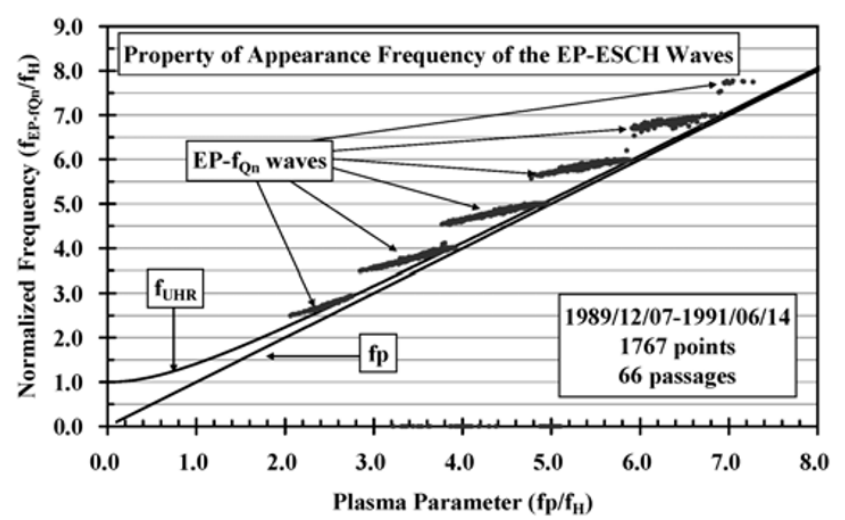

Fig. 6. An $f_{\mathrm{EP}-f_{Q n}} / f_{H}-f_{p} / f_{H}$ diagram of the observed EP- $f_{Q n}$ waves. A total of 1,767 data points were analyzed in the 66 passages of the Akebono satellite inside the plasmasphere. The upper cutoff frequency of the UHR waves and plasma frequency at the satellite observation point are plotted as $f_{\mathrm{UHR}}$ and $f_{p}$, respectively.

3.96-3.98. Therefore, it can be estimated that the wave number of the observed ESCH waves is about $2.45 \mathrm{~m}^{-1}$. Compared with the wave number of the EP- $f_{Q n}$ waves, this ESCH wave number is larger by a factor of 1.35 .

\subsection{Frequency spectra of the EP- $f_{Q n}$ waves}

In order to study on the frequency character of the EP$f_{Q n}$ waves, we have analyzed their frequency ranges and their relation to the local plasma parameters. The data analysis period of the plasma waves is about 2 years - from December 7, 1989 to June 14, 1991. As has already been described in Section 2, the observation frequency range from $20 \mathrm{kHz}$ to $5.2 \mathrm{MHz}$ is covered by dividing it into four frequency swept bands in the PWS system onboard the Akebono satellite. Within the frequency range from $383 \mathrm{kHz}$ to $1,340 \mathrm{kHz}$, the frequency step selected as $7.56 \mathrm{kHz}$ is with a $1-\mathrm{kHz}$ receiving bandwidth. Because the frequency step is larger than that of the bandwidth of the observed EP- $f_{Q n}$ waves, we have selected the EP- $f_{Q n}$ events which appear within a frequency range below $383 \mathrm{kHz}$ in the PWS spectra in order to maintain the accuracy of the frequency analysis. As a first step, by analyzing the upper cutoff frequency of the UHR waves which covers the frequency range from the electron plasma frequency (fp) to the upper hybrid frequency $\left(f_{\mathrm{UHR}}\right)$ in the PWS data, we derived the electron plasma frequency for the observation point of the EP$f_{Q n}$ waves, because fp frequency often becomes difficult to identify in the spectrum due to contamination of the intense $Z$-mode plasma waves. The upper cutoff frequency of the UHR frequency is defined as

$$
f_{\mathrm{UHR}}=\sqrt{f_{p}^{2}+f_{H}^{2}}
$$

where $f_{p}$ and $f_{H}$ indicate the electron plasma and cyclotron frequencies, respectively. The electron cyclotron frequency is calculated from the magnetic field intensity obtained by the MGF detector onboard the Akebono satellite. From Eq. (1), we can obtain the electron plasma frequency.

The occurrence diagram of the observed ESCH waves depending on the normalized plasma frequency $\left(f_{p} / f_{H}\right)$ of the observation point is given in Fig. 6. Within a period from December 7, 1989 to June 14, 1991, we selected

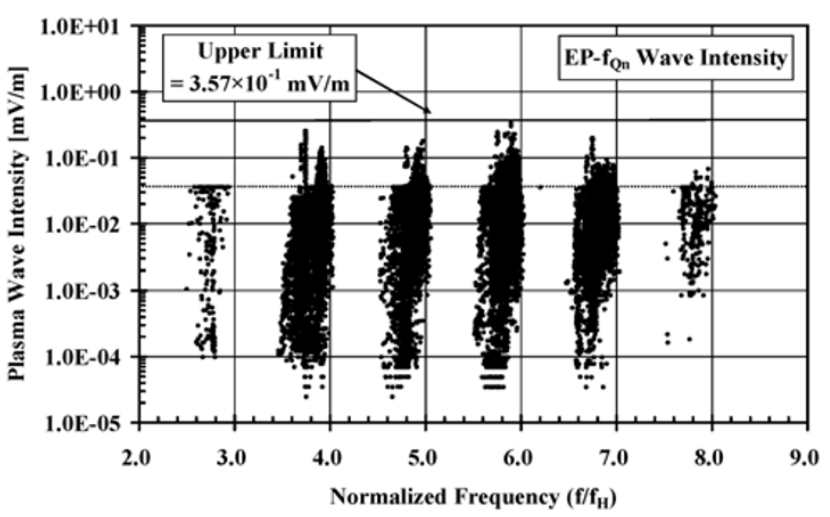

Fig. 7. Dependence of EP- $f_{Q n}$ wave intensity on occurrence frequency normalized by the local electron cyclotron frequency. The upper limit of low gain mode output of the PWS receiver is given by a horizontal solid line [the value of $\left.3.57 \times 10^{-1}(\mathrm{mV} / \mathrm{m})\right]$. The dashed horizontal line indicates the upper limit of the high mode output [the value of $\left.3.57 \times 10^{-2}(\mathrm{mV} / \mathrm{m})\right]$.

the PWS data in which both the EP- $f_{Q n}$ and $f_{D n-m}$ ESCH waves are observed simultaneously, thereby avoiding periods of intense magnetic activities such as major magnetic storms. There are 1,767 selected data points in the 66 passages of the Akebono satellite inside the plasmasphere. The UHR frequency and plasma frequency at the satellite observation point are plotted as $f_{\mathrm{UHR}}$ and $f_{p}$, respectively. In Fig. 6, the ESCH waves satisfy the frequency relation of $(n+1 / 2) f_{H}<f_{\mathrm{EP}-f Q n}<(n+1) f_{H}$ and $f_{\mathrm{UHR}}<f_{\mathrm{EP}-f Q n}$. In each harmonic number $n$, the EP- $f_{Q n}$ waves tend to disappear near the $(n+1) f_{H}$ or $(n+1 / 2) f_{H}$ frequencies, and the waves which belong to the next harmonic branch $(n+1) f_{H}$ appear above the $(n+1 / 2) f_{H}$ frequency. The relation between the observed ESCH waves and plasma frequencies indicates that the ESCH waves appear in the nearest $f_{Q n}$ resonance frequency for the UHR frequency. On the other hand, the ESCH waves are not observed under the condition of the plasma parameter satisfied with less than $f_{p} / f_{H}=2.0$. The $f_{Q n}$ waves observed in the ionosphere (Warren and Hagg, 1968) and magnetosphere (Christiansen et al., 1978) appear in the frequency range of $n f_{H}<f<(n+1) f_{H}$ and $f_{\mathrm{UHR}}<f$. However, in the present observation on results inside the plasmasphere, they are observed only in the limited frequency range, namely, $(n+1 / 2) f_{H}<f_{E S C H}<(n+1) f_{H}$ and $f_{\mathrm{UHR}}<f_{E S C H}$, as shown in Fig. 6. The occurrence of the $\mathrm{ESCH}$ wave frequencies in the plasmasphere indicates that the EP- $f_{Q n}$ waves may be generated due to the temperature anisotropy of electron velocity distribution function as one of the plasma instabilities. Based on the above character of the frequency of occurrence of the ESCH waves in the plasmasphere in comparison to that in the ionosphere and magnetosphere, we named the ESCH waves as EP- $f_{Q n}$ waves.

\subsection{Intensity of EP- $f_{Q n}$ waves}

Figure 7 shows a scatterplot of EP- $f_{Q n}$ waves in terms of normalized frequency versus the intensity. The data numbers are 21,350 events within a period from March 1989 to June 1991 . In this case, the observed data by both the high-gain mode (10,504 events) and the low-gain mode 


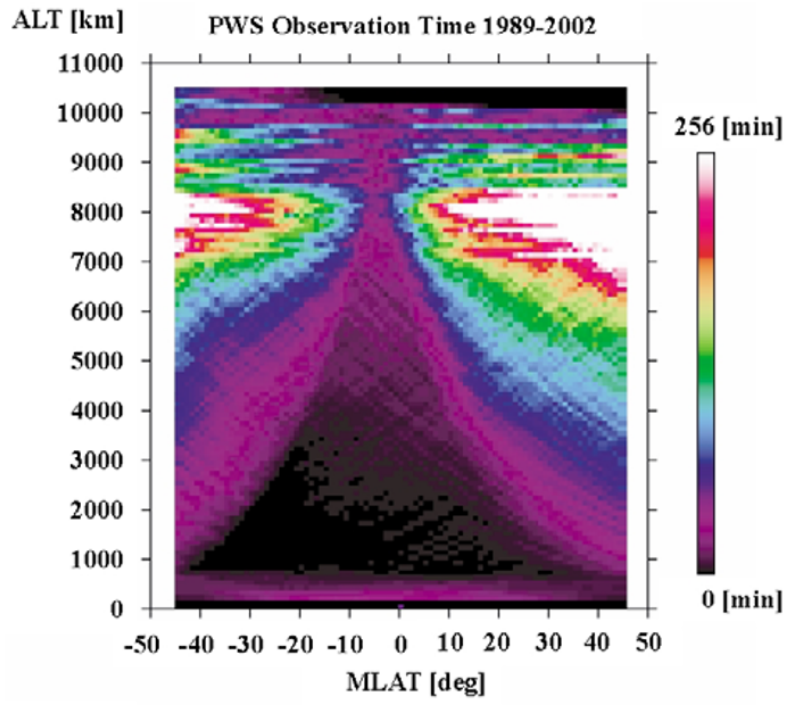

ALT [km] EP- $f_{Q_{n}}$ Occurrence Probability 1989-2002

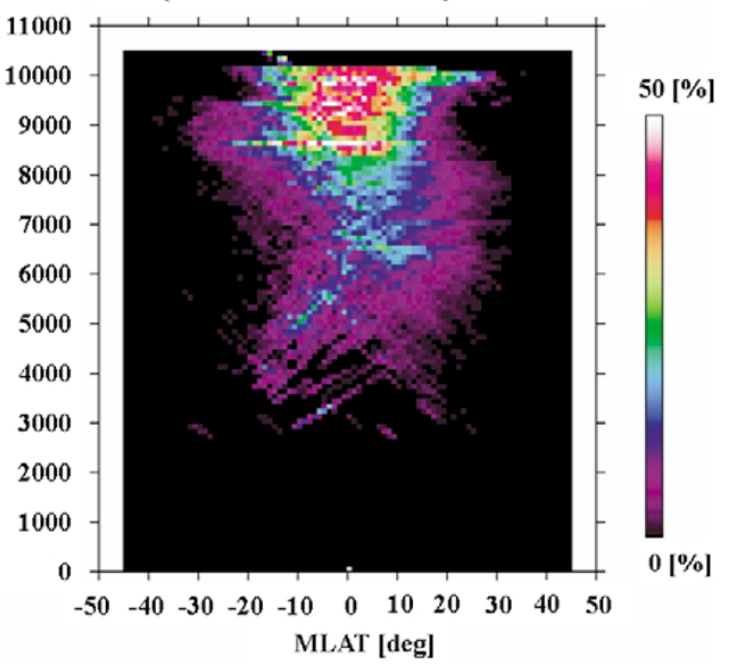

Fig. 8. Temporal coverage of the PWS observation time and occurrence probability of EP- $f_{Q n}$ waves as a function of magnetic latitude $\left( \pm 45^{\circ}\right)$ and altitude of the Akebono satellite observation point. In both panels, the color codes indicate the observation time and occurrence probability, respectively. The EP- $f_{Q n}$ waves are mainly observed near the equatorial region $\left( \pm 10^{\circ}\right)$ of the altitude of $2,500 \mathrm{~km}$ to $10500 \mathrm{~km}$ (satellite apogee).

(10,846 events) are included. The analyzed frequency range is $20-383 \mathrm{kHz}$ because of an improvement in the intensity analysis accuracy. In Fig. 7, the horizontal solid and dashed lines indicate the upper limit levels of the low- and highgain modes of the PWS receiver (values of $3.57 \times 10^{-5}$ $\mathrm{V} / \mathrm{m}$ and $\left.3.57 \times 10^{-4} \mathrm{~V} / \mathrm{m}\right)$, respectively. Interpretation of the spectra of intense plasma wave phenomena must be made with care to avoid any man-made signature due to the saturation of the receiver. In this paper, we took care to avoid false response due to the intense ESCH waves. The intensity of the EP- $f_{Q n}$ waves is within a range of $2.45 \times 10^{-8} \mathrm{~V} / \mathrm{m}$ to $3.31 \times 10^{-4} \mathrm{~V} / \mathrm{m}$. On the other hand, the intensity distribution of the lower harmonic number $n=2$ gives the upper limit value of $3.57 \times 10^{-5} \mathrm{~V} / \mathrm{m}$. The reason for this is that the Akebono satellite provided no chance for the low-gain mode operation of the PWS instrument in the low-latitude plasmasphere region where the plasma parameter is not satisfied with the condition of the EP-
$f_{Q n}$ waves. In each harmonic band, the distribution of the wave intensity is scattered within a frequency range of $(n+1 / 2) f_{H}<f_{\mathrm{EP}-f Q n}<(n+1) f_{H}$; however, the intensity tends to increase weakly as the normalized EP$f_{Q n}$ frequency approaches close to the cyclotron harmonic number. This result suggests that the EP- $f_{Q n}$ waves are easily generated near the electron cyclotron frequency.

\subsection{Occurrence region of the EP- $f_{Q n}$ waves}

In this subsection, the statistical feature of the occurrence region of the EP- $f_{Q n}$ waves is discussed by analyzing all of the plasma wave data within a frequency range of 20 $\mathrm{kHz}$ to $5.1 \mathrm{MHz}$ obtained by the PWS instrument within the time interval between March 1989 and December 2002. The analysis has been restricted to inside the plasmasphere region within the geomagnetic latitude range from $-45^{\circ}$ to $45^{\circ}$. Panel (a) of Fig. 8 shows an observation coverage of the PWS instrument that is plotted as a function of the magnetic latitude and altitude of the Akebono satellite point. The color scale indicates the observation time period for each bin within a range from 0 to $256 \mathrm{~min}$. Each pixel in this figure gives the magnetic latitude and altitude ranges of $1.0^{\circ}$ and $100 \mathrm{~km}$, respectively. Some of the grids within an altitude range of $7,000 \mathrm{~km}$ to $8,500 \mathrm{~km}$ in the northern hemisphere have value of more than $256 \mathrm{~min}$. The coverage is most tenuous in low-altitude and equatorial regions due to the high speed passage of the Akebono satellite near the perigee $(274 \mathrm{~km})$ and lack of the satellite tracking stations. The low coverage region exists within a region of $-20^{\circ}$ to $20^{\circ}$ and below $5000 \mathrm{~km}$.

Panel (b) of Fig. 8 shows the occurrence probability of EP- $f_{Q n}$ waves plotted against the magnetic latitude and altitude of the Akebono satellite. The occurrence probability of the EP- $f_{Q n}$ waves is shown by color scales within a range of $0 \%$ to more than $50 \%$. The grid is the same as that of Panel (a). In Panel (b), EP- $f_{Q n}$ waves appear within the magnetic latitude and altitude regions of the plasmasphere of $-30^{\circ}$ to $30^{\circ}$ and 2,500 to $10,500 \mathrm{~km}$ (satellite apogee), respectively. The EP- $f_{Q n}$ waves were not observed within a low-altitude region of the plasmasphere below $2,500 \mathrm{~km}$ at all. Furthermore, the occurrence probability of the EP- $f_{Q n}$ waves tends to be larger near the equatorial region of the plasmasphere within the magnetic latitude range of $-10^{\circ}$ to $10^{\circ}$. It is also noted that the appearance of the EP- $f_{Q n}$ waves concentrates within the equatorial region of the highaltitude range above $8,500 \mathrm{~km}$. The occurrence probability in this region is about $40 \%$ to more than $50 \%$ of the observation time. It seems that this region is near the slot region of the radiation belt ( $L=2.3-3.5$ ) identified by Walt (1994).

Panel (a) of Fig. 9 shows the plasma wave observation coverage, which is plotted as a function of the magnetic local time and latitude $\left( \pm 45^{\circ}\right)$ of the Akebono satellite observation point. The density of the color scale indicates the coverage time within a range of $0-20 \mathrm{~min}$. Each grid in this panel gives the magnetic local time and latitude ranges of $12 \mathrm{~min}$ and $1.0^{\circ}$, respectively. Some of the grids within the magnetic local time and latitude ranges of 02 to $10 \mathrm{~h}$ and more than $20^{\circ}$ in the northern hemisphere have a value of more than $15 \mathrm{~min}$. The coverage is most tenuous in the equatorial regions of $-10^{\circ}$ to $5^{\circ}$ and the nightside sectors (22-02 MLT) due to the location of the satellite tracking 

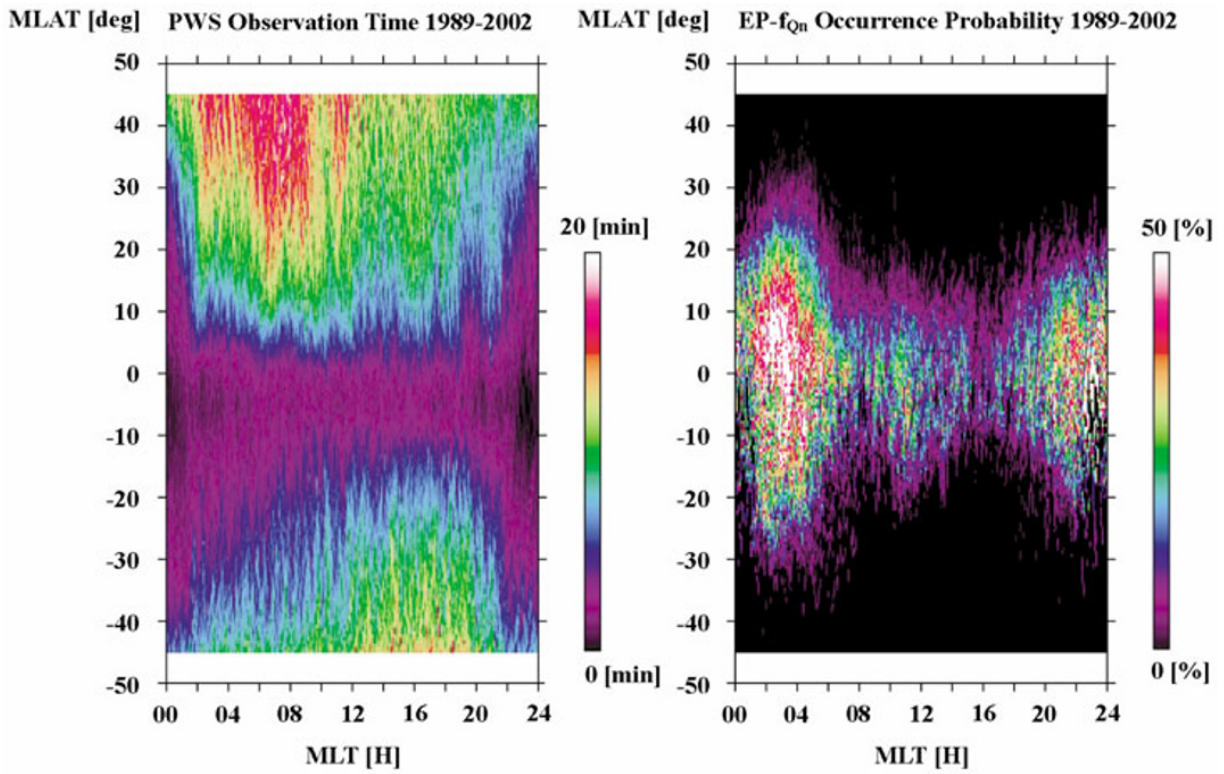

Fig. 9. Temporal coverage of the PWS observation time and occurrence probability of EP- $f_{Q n}$ waves as a function of magnetic local time and latitude $\left( \pm 45^{\circ}\right)$ of the Akebono satellite observation point. The format of the color codes in both panels the same as that of Fig. 8 . The EP- $f_{Q n}$ waves are mainly observed near the equatorial region $\left( \pm 10^{\circ}\right)$ of the plasmasphere in all magnetic local time sectors.

stations.

Panel (b) of Fig. 9 shows the occurrence probability of EP- $f_{Q n}$ waves plotted against the magnetic local time and latitude of the Akebono satellite point. The occurrence probability is indicated by color scales from $0 \%$ to more than $50 \%$, and the grid is the same as that of Panel (a). In this panel, EP- $f_{Q n}$ waves appear in all magnetic local time sectors near the equatorial region of the plasmasphere. The EP- $f_{Q n}$ waves have not been observed within a highlatitude region (MLAT $>35^{\circ}$ and MLAT $\leq 40^{\circ}$ ) of the plasmasphere at all. The observation region of the EP- $f_{Q n}$ waves tends to expand the middle latitude of about $30-40^{\circ}$ in the nightside sectors, compared with that in the dayside sectors. Furthermore, the occurrence probability of the EP$f_{Q n}$ waves also tends to become large in the nightside sectors, while it tends to be small in the dayside sectors of the plasmasphere. The maximum occurrence probability is especially located in the post-midnight sectors (02-04 MLT), with its value of more than $50 \%$, while the minimum occurrence probability is located in the post-noon sectors (14-18 MLT) with its value of less than $15 \%$. The figure shows that the post-midnight sector of the plasmasphere is the most active region of the EP- $f_{Q n}$ waves.

\section{Character of EP- $f_{Q n}$ Occurrence Probability for Solar Activity and Magnetic Disturbances}

\subsection{Solar activity dependence of EP- $f_{Q n}$ waves}

Figure 10 compares the coverage of plasma wave observations and the occurrence probability of EP- $f_{Q n}$ waves during the periods of solar maximum and minimum activities. In this analysis, we determined a total of 9 years separated into two periods-from 1989 to 1993 and from 1999 to 2002 - as solar maximum phase; on the other hand, we determined 5 years of the rest period-from 1994 to 1998 as the solar minimum phase. The solar minimum phase corresponds to the period when the sunspot number is less than
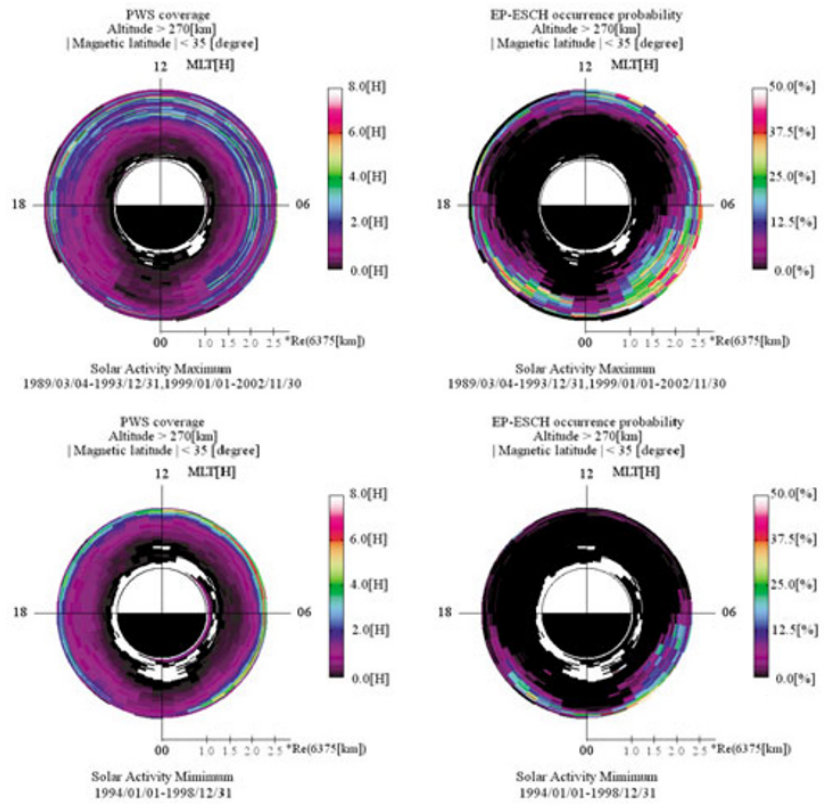

Fig. 10. Temporal coverage of the PWS observation time and occurrence probability of EP- $f_{Q n}$ waves as a function of magnetic local time and altitude of the Akebono satellite observation point within a magnetic latitude of $\pm 35^{\circ}$. The upper and lower panels correspond to solar activities of the maximum and minimum phases, respectively. Occurrence probability of the EP- $f_{Q n}$ waves has a clear dependence on solar activities.

50. The left panel of Fig. 10 gives the plasma wave observation coverage within the magnetic latitude range of $\pm 35^{\circ}$ inside the plasmasphere. Each grid in this figure gives the altitude and magnetic local time ranges of $100 \mathrm{~km}$ and 30 min, respectively. The color codes indicate the coverage time within a range of $0-8 \mathrm{~h}$. The low-altitude grids colored white are grids with no data on the PWS observation. As has already been described in the previous section, the 


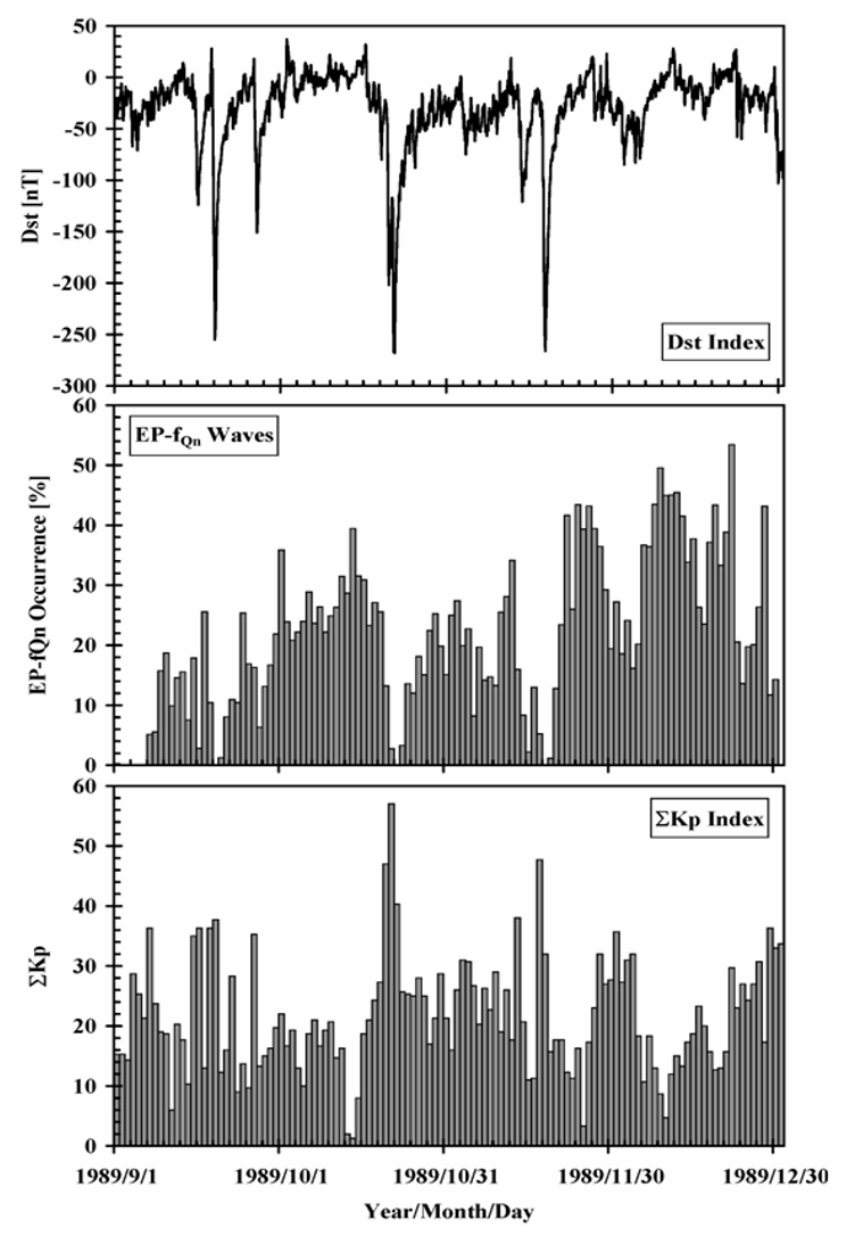

Fig. 11. Dst index, daily EP- $f_{Q n}$ occurrence probability and $\Sigma \mathrm{Kp}$ index within a period from September 1 to December 31, 1989. Occurrence probability of the EP- $f_{Q n}$ waves abruptly decreases during a magnetic storm.

coverage is most tenuous at the low-altitude and nightside sectors (22-02 MLT), while there is a sufficient amount of coverage time at high altitudes of more than $6,000 \mathrm{~km}$ in the local time sector of 02-20 h. Compared with the observation coverage in both periods of solar maximum and minimum phases, there is no significant difference. However, it is noted that the apogee of the Akebono satellite orbit gradually decreases from $10,050 \mathrm{~km}(1990.0)$ to $8350 \mathrm{~km}$ (1995.0) and 6,600 km (2002.0) and becomes lower within a period of the solar minimum phase than that of the solar maximum phase.

The right panel of Fig. 10 shows the probability of EP$f_{Q n}$ occurrence within the periods of solar maximum and minimum phases. Although the character of the EP- $f_{Q n}$ occurrence probability has already been shown in the previous section, in this analysis, the occurrence probability of the EP- $f_{Q n}$ waves in the nightside plasmasphere within the period of solar maximum phase has a large value compared with that in the dayside sector. The maximum occurrence probability is located in the post-midnight sectors (02-05 MLT) and is more than 35-40\%. In these sectors, the EP$f_{Q n}$ waves appear in the low-altitude region near $3,000 \mathrm{~km}$. On the other hand, the minimum occurrence probability is located in the post-noon sectors (14-18 MLT), and in these sectors the EP- $f_{Q n}$ waves appear in the high-altitude region above $7,000 \mathrm{~km}$. The occurrence probability in this region is less than $20 \%$.

Within the period of the solar minimum phase, the occurrence probability of the EP- $f_{Q n}$ has a smaller value compared with that of the solar maximum phase. In particular, the occurrence probability in the dayside sectors (06-18 MLT) indicates a much smaller value of less than $5.0 \%$. In this condition, the majority of the active region of the EP$f_{Q n}$ waves is confined only in the nightside sector. On the other hand, based on the right panel of this figure, it can be seen that the maximum occurrence probability is located in the post-midnight sectors (01-06 MLT). This region is almost the same as that within the period of the solar maximum phase. These results suggest that the activities of the EP- $f_{Q n}$ waves are closely related to solar activity, while the location of the most active region is almost fixed at the postmidnight sectors.

\subsection{Dependence of EP- $f_{Q n}$ occurrence probability on magnetic disturbances}

In order to clarify the relationship between the EP- $f_{Q n}$ wave activities and magnetic disturbances in the magnetosphere, we compared the daily variation in the occurrence probability of the EP- $f_{Q n}$ waves with geomagnetic indices of Dst and Kp provided by WDC-C2, Kyoto University. The first, second, and third panels of Fig. 11 show the Dst index, the daily occurrence probabilities of the EP$f_{Q n}$ waves, and the $\Sigma \mathrm{Kp}$ value, respectively. The period of data analysis is from September 1, 1989 to December 31,1989 . In the upper panel, three intense magnetic storms with an amplitude of more than $200 \mathrm{nT}$ occur within the period of September 18-21, October 20-24, and November 17-20, respectively. Although the $\Sigma \mathrm{Kp}$ value shows an increase of more than 40 during these periods, the EP- $f_{Q n}$ occurrence probability abruptly decreases by less than $10 \%$ or becomes zero for a few days. Moreover, even during the moderate magnetic storm with an amplitude of about 50 $100 \mathrm{nT}$, the EP- $f_{Q n}$ occurrence probability also decreases to about half that value compared with that from within the period of the magnetically quiet condition. This tendency becomes particularly clear during the magnetic storms from November 26 to December 5. On the basis of these results, it can be inferred that the activities of the EP- $f_{Q n}$ waves in the plasmasphere are suppressed by an abrupt decrease of temperature anisotropy of the suprathermal $3-\mathrm{keV}$ electrons due to decrease of the ambient magnetic field intensity in the inner plasmasphere associated with the development of ring current during the main phase of a magnetic storm. In this case, the magnetic moment of the suprathermal electrons is conserved. Based on the RDM data analysis of the Akebono satellite, Nishimura et al. (2005) reported that the temperature anisotropy of high-energy particles $(300 \mathrm{keV}$ to $2.5 \mathrm{MeV}$ ) in the inner magnetosphere dramatically decreases during the main phase of a geomagnetic storm.

\section{Discussion}

\subsection{Quality of the Akebono wave data}

To date, the global character and occurrence feature of ESCH waves have been investigated in the inner magnetosphere $(L=3.5-10)$ by using many experiments of 
plasma wave observations [for example, OGO-5 (Kennel et al., 1970), IMP-6 (Shaw and Gurnett, 1975), S3-A (Anderson and Maeda, 1977), GEOS-1 (Christiansen et al., 1978), ISEE (Kurth et al., 1979), AMPTE (Roeder and Koons, 1989), CRRES (Paranicas et al., 1992)]. Although these satellites may have chance of making plasma wave observations, there have been very few studies on the ESCH waves inside the plasmasphere region, with the exception of reports on the appearance of $(n+1 / 2) f_{H}$ ESCH waves inside the entire plasmasphere region during a large magnetic storm (Oya, 1991). Therefore, it has been accepted that ESCH wave phenomena are not generated inside the plasmasphere (see, for example, Shawhan, 1979). On the other hand, Oya et al. (1981) first identified the appearance of $f_{Q n}$ resonances and sequence of diffuse resonances $\left(f_{D n}\right)$ in the plasmagrams obtained by a stimulated plasma wave experiment onboard the JIKIKEN satellite inside the plasmasphere $(L=1.8-3.1)$. Contrary to most of the previous results, the present analyses of the Akebono satellite plasma wave data reveal that the intense ESCH waves with narrowbanded structure are almost taking place above the UHR frequency in the equatorial regions $\left(-30^{\circ}<\right.$ MLAT $\left.<30^{\circ}\right)$ of the plasmasphere. This contradiction may be due to the lack of plasma wave observation covering the highfrequency range (up to $800 \mathrm{kHz}$ ), with high-frequency resolution and sensitivity inside the plasmasphere region. In this context, the Akebono satellite is the first satellite that makes extremely fine spectra analyses from $20 \mathrm{kHz}$ in the VLF range to $5.1 \mathrm{MHz}$ in $\mathrm{HF}$ range for electric and magnetic components of the plasma waves in the plasmasphere; because the swept frequency analyzers are installed, fine resolution of plasma wave spectra can be obtained with frequency resolution of $0.945 \mathrm{kHz}$ in the range of $20-141 \mathrm{kHz}$ and of $1.89 \mathrm{kHz}$ in that of $141-383 \mathrm{kHz}$ (Oya et al., 1990). Furthermore, because the Akebono satellite has continuously operated more than 14 years, it is possible to examine the long-term and continuous observation data inside the plasmasphere. Consequently, the new results of the present investigations are based on suitable and high-quality data that have not been available in previous studies.

\subsection{Possible generation mechanism of EP- $f_{Q n}$ waves}

Bernstein (1958) investigated the dispersion relation of electrostatic plasma waves for the damping free perpendicular propagation, where the wave vector component parallel to the magnetic field $k_{\|}$is equal to zero. These waves, which are often referred to as "Bernstein modes", can propagate in band of each cyclotron harmonic with cyclic solutions in $\omega-k$ space. The first full solution of the dispersion curves was obtained by Crawford (1965). The feature of the dispersion curve shows a remarkable change that depending on the condition of whether the curve is above or below the UHR frequency, respectively. The general dispersion relation for electrostatic waves in magnetized plasma is described in cylindrical coordinates under the Lorentz gas approximation (Harris, 1959):

$$
\begin{aligned}
1-\frac{2 \pi}{k^{2}} \omega_{p}^{2} \int_{0}^{\infty} d v_{\perp} v_{\perp} \int_{-\infty}^{\infty} d v_{\|} \sum_{n=-\infty}^{\infty} \frac{J_{n}^{2}\left(k_{\perp} v_{\perp} / \Omega\right)}{k_{\|} v_{\|}+n \Omega-\omega} \\
{\left[k_{\|} \frac{\partial f}{\partial v_{\|}}+\frac{n \Omega}{v_{\perp}} \frac{\partial f}{\partial v_{\perp}}\right]=0 }
\end{aligned}
$$

where $\omega_{p}$ is the angular plasma frequency and $\Omega=$ $|e| B / m=2 \pi f_{H}$ is the cyclotron angular frequency. This equation shows that the electron distribution function $f\left(v_{\perp}, v_{\|}\right)$plays a significantly important role. Hereinafter, subscripts $\perp$ and $\|$ denote the perpendicular and parallel components to the magnetic field, respectively. Harris (1959) investigated the role of anisotropic distribution function $f\left(v_{\perp}, v_{\|}\right)$, and found that ESCH waves become unstable under the condition $T_{\perp} / T_{\|} \rightarrow \infty$, which is called the "Harris instability" in this report.

Shima and Hall (1965) carried out analytical studies to determine the frequency range of the instabilities for the finite temperature anisotropy and found the unstable region to be in the frequency range

$$
n+\frac{1}{2}<\frac{\grave{\omega}}{\grave{\Omega}}<n+1-\frac{T_{\|}}{T_{\perp}} .
$$

As has already been described in Section 3.2, the appearance frequency of EP- $f_{Q n}$ waves is satisfied with the frequency relation $(n+1 / 2) f_{H}<f_{\mathrm{EP}-f_{Q n}}<(n+1) f_{H}$, which is almost similar to Eq. (3). Based on this observational fact, it seems that the EP- $f_{Q n}$ waves are generated by temperature anisotropy of the distribution function in suprathermal energy range electrons. Therefore, in the following discussion, we will investigate the validity of the above generation mechanism for two-component plasmas by calculating the plasma wave dispersion relation.

As has already been mentioned in Section 3, since the Akebono satellite does not operate at the low-energy particle observations in the plasmasphere $(L<4.0)$, in order to avoid severe damage to the particle detector due to the radiation belt particles, we can not discuss the electron distribution of the suprathermal energy range based on the simultaneous observations of the plasma waves in this region. Instead of the Akebono satellite observations, statistical observations of the low-energy particles $(100 \mathrm{eV}<$ $E<40 \mathrm{keV})$ in the inner magnetosphere $(2.0<L<7.0)$ by the CRRES satellite were made by Burke et al. (1995). These researchers demonstrated the presence of suprathermal electrons with an energy and temperature of about 3 $\mathrm{keV}$ and $750 \mathrm{eV}$, respectively, in the plasmasphere. The particle distribution, which indicates an abrupt enhancement of the particle fluxes perpendicular to the ambient magnetic field, is a large temperature anisotropy distribution. The temperature anisotropy index $A=T_{\perp} / T_{\|}-1$ is nearly equal to 50 . The ratio of the hot component to background plasma density (nh/nc) is in a range of about $0.01-0.1 \%$ in the usual plasmasphere region. In the present calculation, we used the above ratio of $0.1 \%$. On the other hand, the background particle observations in the plasmasphere by the Cassini satellite (Rymer et al., 2001) showed that the particle distribution is Maxwellian, with a temperature of about $0.5 \mathrm{eV}$. Furthermore, the electron temperature observations by the TED instrument onboard the Akebono satellite used in the present analysis provide a result is almost consistent with that of the Cassini satellite observations. Therefore, based on these observations, we can conclude that the background particle distribution in the plasmasphere can be regarded as a Maxwell distribution.

Figure 12 shows a remarkable frequency of the observed 


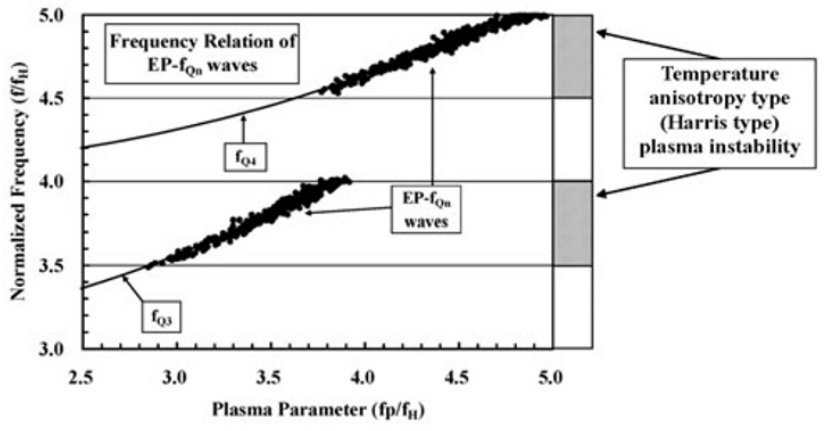

Fig. 12. Comparison between the observed EP- $f_{Q n}$ wave and the $f_{Q n}$ $(n=4,5)$ resonance frequencies calculated from the plasma wave dispersion relation, based on the particle distributions of the background cold and hot components derived from the Cassini and CRRES satellites' observations. The temperatures of the cold and hot components are $0.5 \mathrm{eV}$ and $750 \mathrm{eV}$, respectively. The observed EP- $f_{Q n}$ wave frequency is almost coincident with the calculated $f_{Q n}$ resonance frequency.

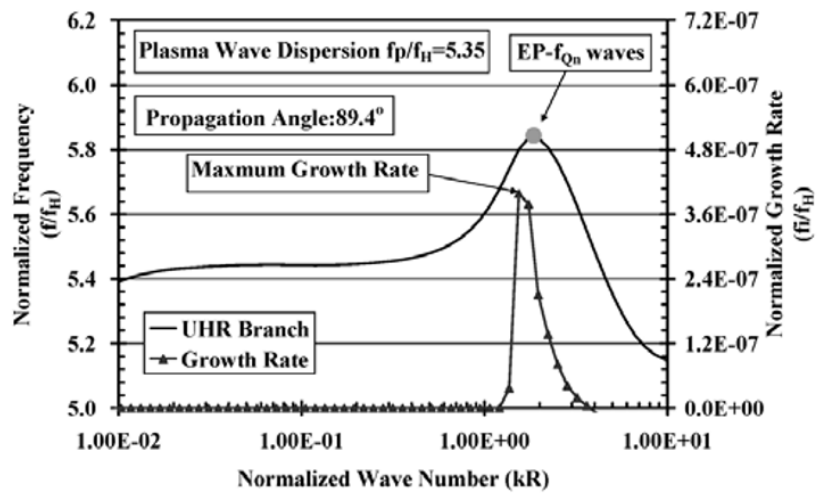

Fig. 13. Calculation results of the dispersion curve of the UHR branch and its growth rate for the multi-component electron distribution. The horizontal and vertical axes give the normalized plasma parameter $\left(f_{p} / f_{H}\right)$ and frequency $\left(f / f_{H}\right)$, respectively. The right vertical axis indicates the growth rate. The gray solid circle presents the observed EP- $f_{Q n}$ wave frequency at 17:47:30 on December 11, 1989.

EP- $f_{Q n}$ waves and the calculated $f_{Q n}$ resonances with group velocity $\mathbf{V} g=0$; these waves belong to the UHR branch with its propagation angle of about $90^{\circ}\left(89.4^{\circ}\right)$ from the Harris type of plasma wave dispersion relation. Here, we calculated the dispersion relation by using the plasma parameters and multi-component plasma distributions at the Akebono observation point at 17:47:30 (UT) on December, 1989. The two gray boxes on the right side of Fig. 12 indicate a frequency region where a temperature anisotropy type (Harris type) plasma instability takes place. This result shows that the occurrence frequencies of the observed EP$f_{Q n}$ waves are almost coincident with those of the $f_{Q n}$ resonances derived from the dispersion relation with the accuracy of $\pm 0.04-0.08 f_{\mathrm{EP}-f_{Q_{n}}} / f_{H}$. The EP- $f_{Q n}$ waves appear within a frequency range of $(n+1 / 2) f_{H}<f<(n+1) f_{H}$ $(n=2,3,4, \ldots)$, corresponding to a frequency region where the Harris-type instability takes place. Moreover, as shown in Fig. 6, EP- $f_{Q n}$ waves are not observed within a plasma parameter $\left(f_{p} / f_{H}\right)$ range of less than 2.0. Based on this point, the observation results of the present study can be well explained, with an assumption of large temper- ature anisotropy of the plasmaspheric hot plasma with its temperature of about $750 \mathrm{eV}$. The dispersion relation of $\mathrm{ESCH}$ waves propagating nearly perpendicular to the magnetic field shows that the frequencies of both Harris instability and $f_{Q n}$ resonance with the lowest harmonic number $(n=1)$ do not overlap with each other when $f_{p} / f_{H}$ is less than 2.0 and the frequency is outside of the UHR branch. That is to say, within the plasma parameter range of less than 2.0, it is shown that $f_{Q n}$ resonance waves are not generated from the Harris instability. On the other hand, $f_{Q n}$ resonance waves which are observed in the ionograms of the Alouette-2 satellite (Warren and Hagg, 1968) cover the frequency range of $n f_{H}<f_{Q n}<(n+1) f_{H}$. This fact indicates that the origin of the $f_{Q n}$ resonances observed by the Alouette- 2 satellite is a RF electric pulse which artificially adds into the magnetized plasma, while the EP- $f_{Q n}$ waves are not observed within a frequency range that can not satisfy the condition of Harris instability. Based on this fact, it can be concluded that the EP- $f_{Q n}$ waves are generated by plasma instability of temperature anisotropy.

\subsection{Propagation direction of EP- $f_{Q n}$ waves for the magnetic field}

As discussed in the previous section, in order to verify the generation mechanism and propagation direction of the EP- $f_{Q n}$ waves in the plasmasphere, we deduced the growth rate of the EP- $f_{Q n}$ waves based on a calculation of the plasma wave dispersion relation by using the multicomponent plasma distributions discussed in Section 5.2 and analyzed spin modulation of the EP- $f_{Q n}$ wave intensity. Figure 13 shows the plasma wave dispersion and growth rate of the UHR branch with its propagation angle of $89.4^{\circ}$ to the ambient magnetic field. Both frequencies are normalized by the electron cyclotron frequency. In this case, the plasma parameter $\left(f_{p} / f_{H}\right)$ was determined from the plasma wave observations in the plasmasphere at 17:47:30 (UT) on December 11, 1989. In Fig. 13, the positive growth rate appears within a normalized wave number range from 1.21 to 3.62, maximizing at the value of 1.54 . The wave number point gives the normalized frequency of the UHR branch as 5.80. The maximum value of the normalized growth rate is $3.98 \times 10^{-7}$. The normalized frequency difference of the observed EP- $f_{Q n}$ waves and the UHR branch derived from the Harris type of the dispersion relation is about 0.04 . Therefore, as has already been discussed in the previous section, the calculation result suggests that the difference is within the accuracy of $\pm 0.04-0.08 f_{\mathrm{EP}-f_{Q n}} / f_{H}$. From this analysis result, it can be inferred that most of the observed EP- $f_{Q n}$ waves are generated close to the almost perpendicular direction $\left(89.4^{\circ}\right)$ to the magnetic field.

Oya (1971) found that the growth rate $(\gamma)$ of the ESCH waves satisfies $\gamma>0$ within the wide range of propagation direction centered around $\theta=60^{\circ}$ for the background hot plasma anisotropy of $T_{\perp} / T_{\|}=5.0$, and confirmed that the instability region also extends in $k$-space, corresponding to the Shima and Hall instability domain for $\omega$. However, from the present calculation results, it is shown that the EP- $f_{Q n}$ waves are probably generated near the almost the perpendicular direction $\left(89.4^{\circ}\right)$ to the magnetic field. This difference between the results of Oya (1971) and the present study possibly comes from the state of particle dis- 


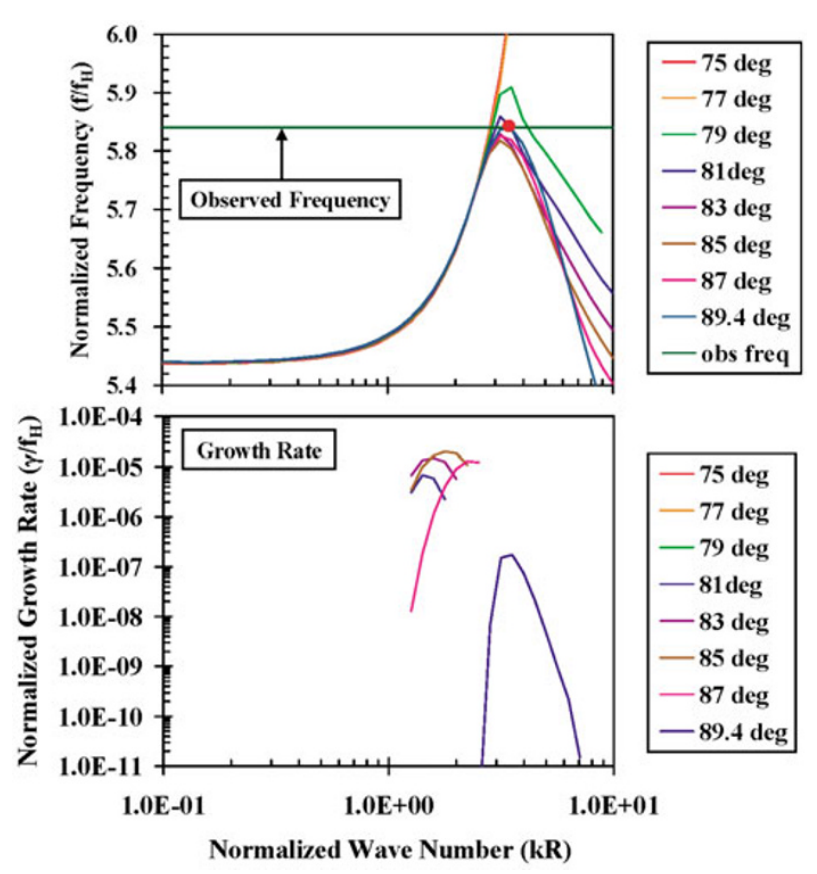

Fig. 14. Calculation results of the dispersion curve of the UHR branch and its growth rate for the propagation angle of $75-89.4^{\circ}$. The red solid circle in the upper panel indicates a point of contact between the frequencies of the observed EP- $f_{Q n}$ waves and of the dispersion curve near the zero group velocity. The positive growth rate appears in a wave number range of more than 1.0 for the propagation angle of more than $81^{\circ}$.

tribution. Oya (1971) used the background cold electron anisotropy model, while in the present study we adapted the multi-component electron distribution in the plasmasphere, based on the satellites' observations. Here, the temperature of the background cold electrons is about $0.5 \mathrm{eV}$, while the perpendicular and parallel temperatures to the magnetic field of the hot electrons are about $750 \mathrm{eV}$ and $14.7 \mathrm{eV}$, respectively. The hot components show a large temperature anisotropy velocity distribution $\left(T_{\perp} / T_{\|}>32.0\right)$, based on the CRRES satellite observations of the pitch angle distribution (Burke et al., 1995). In the above discussion, the positive growth rate appears near the point of $V g=0$ which belongs to the UHR branch with its propagation angle of $89.4^{\circ}$ for the above plasma parameter. Here, we also investigate the characteristics of the occurrence frequency and positive growth rate of the EP- $f_{Q n}$ waves for other propagation angle. The upper and bottom panels in Fig. 14 show the calculation results of the plasma wave dispersion relation and the growth rate belonging to the UHR branch, respectively. In this case, as shown in the upper panel, only the propagation angle of $89.4^{\circ}$ indicates the consistency of the observed EP- $f_{Q n}$ wave frequency at 17:47:30 (UT) on December 11, 1989 with the frequency near $V g=0$. This result suggests that the EP- $f_{Q n}$ waves observed in the plasmasphere propagate to almost the perpendicular direction in the ambient magnetic field. On the other hand, as shown in the bottom panel in Fig. 14, the positive growth rate appears for the large propagation angle of more than $81^{\circ}$ in the wave number range of more than 1.0. This means that the ESCH waves belonging to the UHR branch can not grow in the small propagation angle for the present plasma model.

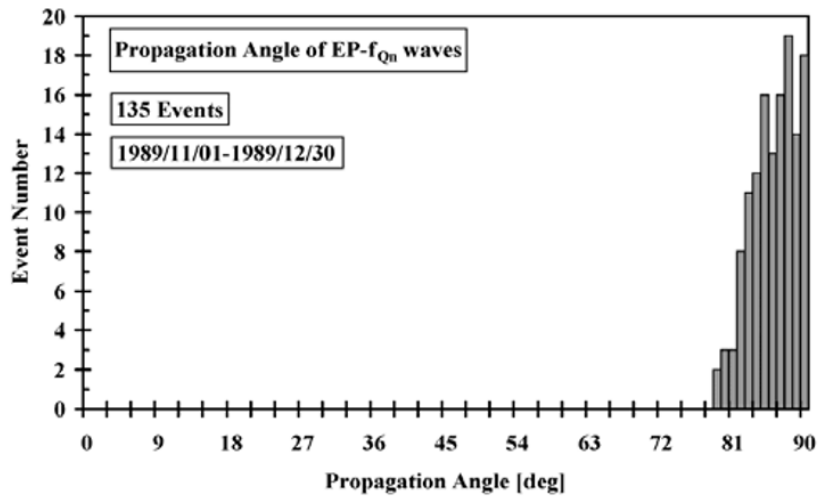

Fig. 15. Histogram of the distribution of the propagation angle of the EP- $f_{Q n}$ waves for 135 events, which are observed within a period from November to December 1989. The horizontal and vertical axes give the propagation angle and event number, respectively. The distribution shows that majority of the $135 \mathrm{EP}-f_{Q n}$ waves concentrate on the large propagation angle of more than $79^{\circ}$.

Furthermore, the present calculation result indicates that the maximum growth rate appears near the observed frequency only in the case of the propagation angle of $89.4^{\circ}$ although the positive growth rate does exist for the propagation angle of $81-87^{\circ}$. Therefore, based on the results of the present calculation of the plasma wave dispersion relation, it can be suggested that the EP- $f_{Q n}$ waves in the plasmasphere are generated near the large propagation angle due to the temperature anisotropy instability.

In order to verify the evidence that the EP- $f_{Q n}$ waves almost propagate to the perpendicular direction in the ambient magnetic field, we investigated the propagation direction of EP- $f_{Q n}$ waves by analyzing the spin modulation of the plasma wave intensity due to the satellite rotation. Figure 15 shows a histogram of propagation angle of the EP- $f_{Q n}$ waves to the ambient magnetic field for 135 events within a period from November 1 to December 31, 1989. In this case, the magnetic field vector is determined from the MGF observation data. In Fig. 15, the distribution of the propagation direction of the EP- $f_{Q n}$ waves concentrates only on a large propagation angle of more than $79^{\circ}$. The tendency of the distribution indicates that the event number abrupt increases with increase of the propagation angle. Therefore, based on the above observational results, it can be concluded that majority of the observed EP- $f_{Q n}$ waves propagate nearly in the perpendicular direction to the magnetic field.

From the above results, the EP- $f_{Q n}$ waves may be generated by suprathermal electrons $(>750 \mathrm{eV})$ with large temperature anisotropy velocity distribution $\left(T_{\perp} / T_{\|} \cong 50.0\right)$ and propagate with the propagation angle of about $80-89^{\circ}$ for the magnetic field. Following propagation in the earth's magnetic field of the plasmasphere, the propagation angle varies due to variations in the plasma density and magnetic field intensity. Then, only the EP- $f_{Q n}$ waves with the propagation angle close to $90^{\circ}$ can survive for a long time, while the EP- $f_{Q n}$ waves with other propagation angle disappear rapidly due to the strong damping. Therefore, only the EP$f_{Q n}$ waves with the propagation angle of about $90^{\circ}$ are observable in the plasmasphere by the Akebono satellite. The 


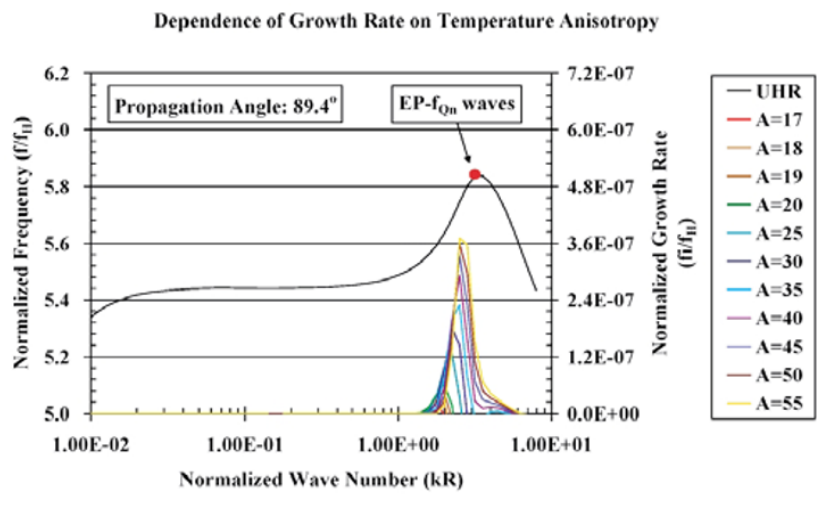

Fig. 16. Dependence of growth rate on temperature anisotropy of the EP- $f_{Q n}$ waves for the propagation angle of $89.4^{\circ}$. The color curves indicate the growth rate normalized by the electron cyclotron frequency for the temperature anisotropy index up to 55. The plasma parameter is the same as that in Fig. 13. The positive growth rate appears near the wave number region of the observed EP- $f_{Q n}$ waves for the temperature anisotropy index of more than $A=40$.

detail propagation effect of the EP- $f_{Q n}$ waves should be solved in future studies.

\subsection{Requirement of the large temperature anisotropy of hot electron distribution}

In this section, we discuss the requirement of the large temperature anisotropy of the hot electron component related to the generation of the EP- $f_{Q n}$ waves in the lowlatitude plasmasphere. In order to clarify the requirement of the hot electron component, we calculated the growth rate of the plasma waves by solving the plasma wave dispersion relation for the two-component electron distributions. Figure 16 shows the growth rate dependence of the EP- $f_{Q n}$ waves on the temperature anisotropy index in a range up to $A=55$ for the propagation angle of $89.4^{\circ}$, based on the above calculation and observation results. In this case, it is shown that the positive growth rate of the EP- $f_{Q_{n}}$ waves appears in the normalized wave number region of more than 1.0 only when the temperature anisotropy index is a large value of more than 17. Moreover, the wave number region indicates well enough that the large growth rate tends to approach the observed EP- $f_{Q n}$ wave number. Therefore, this result of the present temperature anisotropy model implies that the large temperature anisotropy of more than 40 must be required for the generation of the EP- $f_{Q n}$ wave propagating near the perpendicular direction to the magnetic field. On the other hand, the CRRES particle observations (Burke et al., 1995) showed that the flux distribution of hot electrons with $3-\mathrm{keV}$ energy in the equatorial region of the inner plasmasphere $(L=2.6)$ concentrates in the pitch angle range from $80-90^{\circ}$. The peak magnitude of the particle flux was located near the perpendicular direction of the magnetic field. Therefore, the temperature anisotropy index derived from the pitch angle distribution gives a large value of more than 32. From this result, the hot electrons indicate a large temperature anisotropy distribution in the low-latitude plasmasphere where the EP- $f_{Q n}$ waves are frequently observed by the Akebono satellite. However, the temperature anisotropy index is slightly smaller than that inferred from the calculation results of the plasma wave dispersion rela-

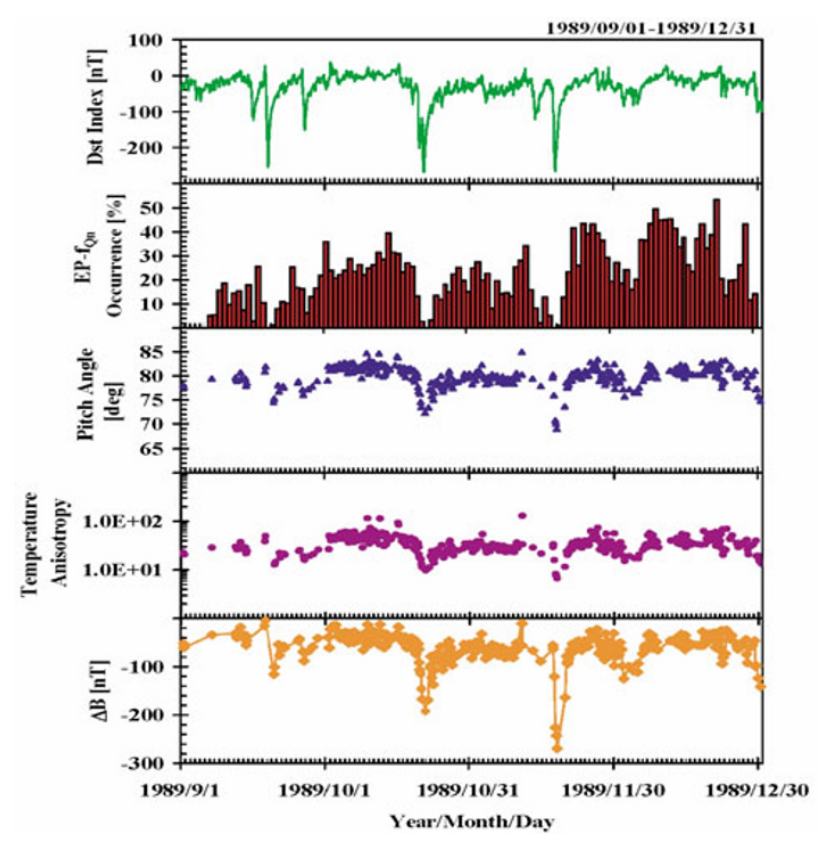

Fig. 17. A summary plot of the Dst index, daily occurrence probability of the EP- $f_{Q n}$ waves, pitch angle of $3-\mathrm{keV}$ electrons and temperature anisotropy and magnetic field deviation at the magnetic equator from the IGRF90 model field within a period from September 1 to December 31. The pitch angle of the $3-\mathrm{keV}$ electrons and temperature anisotropy near the magnetic equatorial region are derived from the magnetic field intensity in the plasmasphere obtained from the MGF instrument. The initial values of the pitch angle and temperature anisotropy are 820 and 50.6, respectively, at 19:07 (UT) on October 1. The magnetic field intensity at this time was $2071 \mathrm{nT}$ at the altitude of $9,000 \mathrm{~km}$. A clear decrease of the pitch angle of the electrons and temperature anisotropy can be seen together with a decrease in the occurrence probability of the EP- $f_{Q n}$ waves associated with geomagnetic storms.

tion of the UHR branch. This inconsistency probably indicates that the simultaneous observations of both the plasma waves and particles are not operated in the same condition of the plasmaspheric plasmas. In the future, we need to operate these simultaneous observations in the inner plasmasphere region by the next satellite mission.

\subsection{Causes of the suppression of the EP- $f_{Q n}$ wave ac- tivities during geomagnetic disturbances}

We have shown that the activities of the EP- $f_{Q n}$ waves in the plasmasphere tend to be abruptly suppressed during geomagnetic disturbances. This main cause seems to be due to an abrupt decrease in the temperature anisotropy of the suprathermal electrons in this region, and these suprathermal electrons are related to the generation of the EP- $f_{Q n}$ waves. From the calculation result of plasma dispersion relation in the previous discussion, the large temperature anisotropy of more than 40-50 is necessary to generate the EP- $f_{Q n}$ waves at the observed frequency. Then, this decrease in the temperature anisotropy of the electrons in the plasmasphere can be explained by the adiabatic cooling process due to the decrease of the ambient magnetic field intensity associated with the development of ring current during the main phase of geomagnetic storms.

In order to verify the importance of the adiabatic cooling process to the EP- $f_{Q n}$ wave activities associated with an abrupt decrease of the ambient magnetic field intensity due to the development of ring current in the inner mag- 
netosphere, we investigated the variations in the pitch angle of the suprathermal $3-\mathrm{keV}$ electrons and temperature anisotropy using the magnetic field data near the magnetic equator obtained from the MGF instrument onboard the Akebono satellite. The variations in the pitch angle distribution can be obtained from the conservation of the magnetic moment of the electrons as shown in the following equation:

$$
\mu=\frac{m v^{2} \sin ^{2} \alpha_{e q}}{2 B}
$$

where $\alpha_{e q}$ is the pitch angle at the magnetic equator. From the conservation of the magnetic moment when the ambient magnetic field intensity changes from $B_{1}$ to $B_{2}$ near the magnetic equator, the pitch angle variations at this point are given as

$$
\alpha_{e q 2}=\sin ^{-1}\left(\sqrt{\frac{B_{2}}{B_{1}}} \sin \alpha_{e q 1}\right) .
$$

On the other hand, the relationship between the electron temperature and energy is represented by the following equations

$$
\begin{aligned}
& \frac{1}{2} m v_{\perp}^{2}=\frac{1}{2} m v^{2} \sin ^{2} \alpha_{e q}=k_{B} T_{\perp} \\
& \frac{1}{2} m v_{\|}^{2}=\frac{1}{2} m v^{2} \cos ^{2} \alpha_{e q}=k_{B} T_{\|}
\end{aligned}
$$

where $k_{B}, T_{\perp}$, and $T_{\|}$represent the Boltzman constant, the electron temperatures perpendicular and parallel to the ambient magnetic field, respectively. From the Eq. (7), we obtain the relationship between the temperature anisotropy $\mathrm{A}$ and the electron pitch angle

$$
A=\frac{T_{\perp}}{T_{\|}}-1=\tan ^{2} \alpha_{e q}-1 .
$$

Here, since the initial values of the pitch angle, temperature anisotropy, and magnetic field intensity are necessary to calculate the variations in these parameters, we determined the initial values of the parameters as $82^{\circ}, 50.6$, and $2071 \mathrm{nT}$, respectively, based on the calculation results of the plasma wave dispersion relation shown in the previous section. On the other hand, we identified the start time of the present calculations of the pitch angle and temperature anisotropy as 19:07 (UT) on October 1, 1989. The initial value of the $\mathrm{t}$ magnetic field intensity of $2071 \mathrm{nT}$ is obtained at the altitude of $9000 \mathrm{~km}$ at the magnetic equator of the inner plasmasphere at $L=2.6$. Moreover, we adapted the initial values of the pitch angle and temperature anisotropy index as $82^{\circ}$ and $A=50$ at this point, respectively.

Figure 17 shows a summary plot of the Dst index, daily occurrence probability of the EP- $f_{Q n}$ waves, pitch angle of 3-keV electrons, and temperature anisotropy and magnetic field deviation at the magnetic equator from the IGRF model field within the period from September 1 to December 31. The pitch angle of the 3-keV electrons and temperature anisotropy near the magnetic equatorial region are derived from the magnetic field intensity in the plasmasphere obtained from the MGF instrument. As has already been mentioned in the previous section, the second panel in Fig. 17 shows that the occurrence probability of the EP- $f_{Q n}$ waves abruptly decreases during a high geomagnetic activity. On the other hand, the variations of the pitch angle distribution of the $3-\mathrm{keV}$ electrons in the plasmasphere show an abrupt decrease from $82^{\circ}$ to $70^{\circ}$ during the main phase of each geomagnetic storm event. Furthermore, as shown in the bottom panel in Fig. 16, the temperature anisotropy also extremely decreases from 50.6 to around 10 in the period when the magnetic field deviation shows a negative value of less than $-100 \mathrm{nT}$ in the equatorial and low-latitude regions of the plasmasphere. It is interesting that the minimum value of the magnetic field deviation is not consistent with the value of the Dst index within a period from September to October when the Akebono satellite apogee was located in the pre-noon sector. Therefore, this result implies that the intensity of the ring current in the inner magnetosphere has a strong dependence on magnetic local time, which indicates that the intensity tends to be weak in the dayside sector. This evidence of ring current distribution has been well-known in the previous works (e.g., Daglis et al., 1999). During the recovery phase of each storm event, the occurrence probability of the EP- $f_{Q n}$ waves, pitch angle distribution, and temperature anisotropy recover to the level of the magnetically quiet condition within a period of 2-3 days. These results suggest that the major process of the suppression of the EP- $f_{Q n}$ wave activities in the plasmasphere during the geomagnetic storms is the adiabatic cooling process associated with an abrupt decrease of the ambient magnetic field intensity due to the development of the ring current in the inner magnetosphere.

It is interesting that the variations of the pitch angle distribution and temperature anisotropy in Fig. 17 indicate that they do not completely recover to those during the prestorm phase. This is particularly evident in the geomagnetic storm event which occurred on October 20-22, 1989; the characteristic tendency of the variations of these parameters is clearly shown in Fig. 17. This result suggests that the structure and distribution of the ambient magnetic field in the inner magnetosphere do not completely recover to the level of the pre-storm condition: that is, the ring current formed in the inner magnetosphere during the large geomagnetic storms is not completely decayed due to the loss processes of the charge exchange and atmospheric precipitation associated with wave-particle interaction.

\section{Conclusion}

Electrostatic electron cyclotron harmonic (ESCH) waves are often observed in the low-latitude region of the plasmasphere by the plasma waves and sounder experiment (PWS) on board the Akebono satellite carried out from March 1989 to December 2002. We investigated the occurrence region, the spectral characteristics, the dependence of solar activity and geomagnetic activity, and the generation mechanism of the ESCH waves. In the present study, the ESCH wave phenomena have been named as the equatorial plasmaspheric $f_{Q n}$ resonance waves (abbreviated EP- $f_{Q n}$ waves). The statistical results of the present study are summarized as follows:

1. EP- $f_{Q n}$ waves mainly appear within an altitude range from $3000 \mathrm{~km}$ to the apogee of the satellite (about 
$10,500 \mathrm{~km}$ ) and are confined to the low-latitude region of the plasmasphere within a magnetic latitude range from $-30^{\circ}$ to $30^{\circ}$.

2. EP- $f_{Q n}$ waves are observable in quiet or moderate geomagnetic activity of the plasmasphere in all of the magnetic local time sectors of the plasmasphere. The occurrence probability shows a clear enhancement in the early morning sector of 01-03 MLT in the plasmasphere, while it gives the minimum value in the dayside sector of 14-18 MLT.

3. EP- $f_{Q n}$ waves appear above the UHR frequency at the lowest harmonic number branch of the $f_{Q n}$ resonances with satisfying the relationship $(n+1 / 2) f_{H}<f_{Q n}<$ $(n+1) f_{H}$. The spectra are characterized by a narrowband structure with a relative bandwidth of $\delta f / f<$ $3.0 \times 10^{-3}$. The wave intensity is in the of $2.45 \times 10^{-8}$ to $3.31 \times 10^{-4} \mathrm{~V} / \mathrm{m}$.

4. The plasma wave activities are abruptly suppressed within a period of strong magnetic disturbances compared with those of the quiet condition of the plasmasphere. The suppression of the EP- $f_{Q n}$ wave activities in the plasmasphere during the magnetic disturbances is due to an abrupt decrease of temperature anisotropy of the suprathermal electrons by a decrease of the ambient magnetic field intensity in the inner plasmasphere associated with the development of ring current during the main phase of a geomagnetic storm.

5. The activities of the EP- $f_{Q n}$ waves tend to increase during the solar maximum phase, compared with those during the solar minimum phase. In general, it seems that the seed electrons of the EP- $f_{Q n}$ waves are injected from the nightside plasmasheet into the inner plasmasphere under the action of the large-scale intense electric field during geomagnetic storms (e.g., Shinbori et al., 2005), keeping the first adiabatic invariant. Such strong disturbances tend to occur during solar the maximum phase more frequently than during the solar minimum phase. Therefore, since the amount of the seed electrons tend to increase in the inner plasmasphere during the solar maximum phase, the occurrence probability of the EP- $f_{Q n}$ waves tends to increase during this period, compared with that during the solar minimum phase.

6. The majority of the EP- $f_{Q n}$ waves seem to propagate in the direction perpendicular to the ambient magnetic field based on our analysis result of the spin modulation of the EP- $f_{Q n}$ wave intensity.

Based on the analysis of the dispersion relation of the EP- $f_{Q n}$ waves, supra-thermal plasma with the energy and temperature of $3 \mathrm{keV}$ and $750 \mathrm{eV}$, respectively, with high temperature anisotropy $(A=50)$ observed by the CRRES satellite (Burke et al., 1995) is likely to generate the EP- $f_{Q n}$ waves in the plasmasphere. Therefore, it is concluded that the appearance of the EP- $f_{Q n}$ waves revealed a more active and turbulent nature of the plasmaspheric plasma than it has been recognized to date.

Acknowledgments. The Akebono (EXOS-D) satellite was established by the Institute of Space and Astronautical Science (ISAS). The authors thank Dr. Nagatsuma for providing the science data of MGF and for valuable discussions. The Dst and Kp indices were provided from WDC-C2 Kyoto University. Efforts of 3 authors (T. Ono, M. Iizima, and A. Kumamoto) are supported by the 21st century COE program of the Ministry of Educations Culture Sports, Science and Technology, Japan. One of the authors (A. Shinbori) is supported by a grant of Research Fellowship of the Japan Society for the Promotion of Science for Young Scientist.

\section{References}

Abe, T., K. Oyama, H. Amemiya, S. Watanabe, T. Okuzawa, and K. Schlegel, Measurements of temperature and velocity distribution of thermal electrons by the Akebono (EXOS-D) satellite-Experimental setup and preliminary results-, J. Geomag. Geoelectr., 42, 537-554, 1990.

Anderson, R. A. and K. Maeda, VLF emissions associated with enhanced magnetospheric electrons, J. Geophys. Res., 82, 135-146, 1977.

Ashour-Abdalla, M. and C. F. Kennel, Nonconvective and convective electron cyclotron harmonic instabilities, J. Geophys. Res., 83, 1531-1543, 1978.

Bernstein, I. B., Waves in a plasma in a magnetic field, Phys. Rev., 109, 10-21, 1958.

Birmingham, T. J., J. K. Alexander, M. D. Desch, R. F. Hubbard, and B. M. Pedersen, Observations of electron gyroharmonic waves and the structure of the Io torus, J. Geophys. Res., 86, 8497-8507, 1981.

Burke, W. J., A. G. Rubin, D. A. Hardy, and E. G. Holeman, Banded electron structures in the plasmasphere, J. Geophys. Res., 100, 7759$7769,1995$.

Christiansen, P., P. Gough, G. Martelli, J.-J. Bloch, N. Cornilleau, J. Etcheto, R. Gendrin, D. Jones, C. Beghin, and P. Dercreau, Geos I: Identification of natural magnetospheric emissions, Nature, 272, 682-686, 1978

Crawford, F. W., A review of cyclotron phenomena in plasma, Nucl. Fusion, 5, 73, 1965.

Curtis, S. A. and C. S. Wu, Electrostatic and electromagnetic gyroharmonic emissions due to energetic electrons in magnetospheric plasmas, J. Geophys. Res., 84, 2057-2075, 1979.

Daglis, I. A., R. M. Thorne, W. Baumjohann, and S. Orsini, The terrestrial ring current: Origin, formation, and decay, Rev. Geophys., 37, 407-438, 1999.

Fredricks, R. W., Plasma instability at $(n+1 / 2) f c$ and its relationship to some satellite observations, J. Geophys. Res., 76, 5344-5348, 1971

Fredricks, R. W. and F. L. Scarf, Recent studies of magnetospheric electric field emissions above the electron gyrofrequency, J. Geophys. Res., 78, 310-314, 1973.

Fukunishi, H., R. Fujii, S. Kokubun, K. Hayashi, T. Tohyama, Y. Tonegawa, S. Okano, M. Sugiura, K. Yumoto, I. Aoyama, T. Saito, T. Iijima, A. Nishida, and M. Natori, Magnetic field observations on the Akebono (EXOS-D) satellite, J. Geomag. Geoelectr., 42, 385-409, 1990.

Harris, E. G., Unstable plasma oscillations in a magnetic field, Phys. Rev. Lett., 2, 34-36, 1959.

Horne, R. B., R. M. Thorne, N. P. Meredith, and R. R. Anderson, Diffuse auroral electron scattering by electron cyclotron harmonic and whistler mode waves during an isolated substorm, J. Geophys. Res., 108, 1290, doi:10.1029/2002JA009736, 2003.

Hubbard, R. F. and T. J. Birmingham, Electrostatic emissions between electron gyroharmonics in the outer magnetosphere, J. Geophys. Res., 83, 4837-4850, 1978 .

Kennel, C. F., F. L. Scarf, R. W. Fredricks, J. H. McGehee, and F. V. Coroniti, VLF electric field observations in the magnetosphere, J. Geophys. Res., 89, 3015-3018, 1970.

Koons, H. C., B. C. Edgar, J. F. Fennell, and D. J. Georney, Observations of electron cyclotron harmonic emissions associated with field-aligned electron beams, J. Geophys. Res., 92, 7531-7537, 1987.

Kurth, W. S., J. D. Craven, L. A. Frank, and D. A. Gurnett, Intense electrostatic waves near the upper hybrid resonance frequency, J. Geophys. Res., 84, 4145-4164, 1979.

LaBelle, J., D. R. Ruppert, and R. A. Treumann, A statistical study of banded magnetospheric emissions, J. Geophys. Res., 104, 293-303, 1999.

Lyons, L. R., Electron diffusion driven by magnetospheric electrostatic waves, J. Geophys. Res., 79, 575-580, 1974.

Matsumoto, H. and H. Usui., Intense bursts of electron cyclotron harmonic waves near the dayside magnetopause observed by GEPTAIL, Geophys. Res. Lett., 24, 49-52, 1997. 
Nelms, G. L. and G. E. K. Lockwood, Early results from the topside sounder in the Alouette II satellite, Space Res., 7, 604-623, 1966.

Nishimura, Y., A. Shinbori, T. Ono, M. Iizima, A. Kumamoto, and A. Morioka, Evolution of pitch angle distributions of relativistic electrons in the radiation belt during magnetic storms, J. Geophys. Res., 2005 (submitted).

Oya, H., Sequence of diffuse plasma resonances observed on Alouette 2 ionograms, J. Geophys. Res., 75, 4279-4285, 1970.

Oya, H., Verification of theory on weak turbulence relating to the sequence of diffuse plasma resonances in space, Phys. Fluids, 14, 2487-2499, 1971.

Oya, H., Plasma flow hypothesis in the magnetosphere relating to frequency shift of electrostatic plasma waves, J. Geophys. Res., 80, 27832789, 1975.

Oya, H., Studies on plasma and plasma waves in the plasmasphere and auroral particle acceleration region, by PWS on board the EXOS-D (Akebono) satellite, J. Geomag. Geoelectr., 43, 369-393, 1991.

Oya, H., T. Ono, and T. Kamada, Stimulation of plasma waves in the magnetosphere using satellite JIKIKEN (EXOS-B), Part I, Observation of plasma resonances, J. Geomag. Geoelectr., 33, 3-25, 1981.

Oya, H., A. Morioka, K. Kobayashi, M. Iizima, T. Ono, H. Miyaoka, T. Okada, and T. Obara, Plasma wave observation and sounder experiments (PWS) using the Akebono (EXOS-D) satellite-instrumentation and initial results including discovery of the high altitude equatorial plasma turbulence, J. Geomag. Geoelectr., 42, 411-422, 1990.

Oya, H., M. Iizima, and A. Morioka, Plasma turbulence disc circulating the equatorial region of the plasmasphere identified by the plasma wave detector (PWS) onboard the Akebono (EXOS-D) satellite, Geophys. Res. Lett., 18, 329-332, 1991.

Paranicas, C., W. J. Hughes, H. J. Singer, and R. R. Anderson, Banded electrostatic emissions observed by the CRRES plasma wave experiment, $J$. Geophys. Res., 97, 13889-13898, 1992.

Roeder, J. L. and H. C. Koons, A survey of electron cyclotron waves in the magnetosphere and the diffuse auroral electron precipitation, $J$.
Geophys. Res., 94, 2529-2541, 1989.

Roeder, J. L., V. Angelopoulos, W. Baumjohann, and R. R. Anderson, Observations of correlated broadband electrostatic noise and electron cyclotron emissions in the plasma sheet, Geophys. Res. Lett., 18, 5356, 1991.

Rymer, A. M., A. J. Coates, K. Svenes, G. A. Abel, D. R. Linder, B. Narheim, M. Thomsen, and D. T. Young, Cassini plasma spectrometer electron spectrometer measurements during the Earth swing-by on August 18, 1999, J. Geophys. Res., 106, 30177-30198, 2001.

Shaw, R. L. and D. A. Gurnett, Electrostatic noise bands associated with the electron gyrofrequency and plasma frequency in the outer magnetosphere, J. Geophys. Res., 80, 4259-4271, 1975.

Shawhan, S. D., Magnetospheric plasma wave research 1975-1978, Reviews of Geophys. And Space Phys., 17, 705-724, 1979.

Shima, Y. and L. S. Hall, Electrostatic instabilities in a plasma with anisotropic velocity distribution, Phys. Rev., 139, A1115-A1116, 1965.

Shinbori, A., Y. Nishimura, T. Ono, M. Iizima, A. Kumamoto, and H. Oya, Electrodynamics in the duskside inner magnetosphere and plasmasphere during a super magnetic storm on March 13-15, 1989, Earth Planets Space, 57, 643-659, 2005.

Walt, M., Introduction to Geomagnetically Trapped Particle, Cambrige Unversity Press, Cambrige, 1994.

Warren, E. S. and E. L. Hagg, Observation of electrostatic resonances of the ionospheric plasma, Nature, 220, 466-468, 1968.

Young, T. S. T., J. D. Callen, and J. E. McCune, High-frequency electrostatic waves in the magnetosphere, J. Geophys. Res., 78, 1082-1099, 1973.

A. Shinbori (e-mail: shinbori@stelab.nagoya-u.ac.jp), T. Ono, M. Iizima, A. Kumamoto, S. Shirai, A. Hanaoka, K. Okamoto, M. Ohashi, and H. Oya 